\title{
THE PROPAGATION OF SMALL AMPLITUDE LONG WAVES ON THE SURFACE OF SUPERFLUID HELIUM
}

\author{
R. S. JOHNSON ${ }^{1}$
}

(Received 5 October 1982)

\begin{abstract}
Various problems related to the propagation of small amplitude long waves on the surface of superfluid helium (helium II), usually called third sound, are studied on the basis of the appropriate governing equations. The two-fluid continuum model due to Landau is considered, with the effects of healing and relaxation incorporated, and viscosity, heat conduction and compressibility terms retained. The helium vapour is treated as a classical (Newtonian) compressible gas and the exact jump conditions across the liquid/vapour interface are employed. These liquid, vapour and jump equations constitute the exact problem although, in an effort to reduce the complexity of the equations, a simplified set of 'model' surface boundary conditions is also introduced. This full set of equations is non-dimensionalised taking care that all physical parameters are defined using only the undisturbed depth of the layer as the appropriate length scale. The ratio depth/wavelength $(\delta)$ is then a separate parameter as is the wave amplitude/depth ratio $(\varepsilon)$. The limit which corresponds to the wave under discussion is then $\varepsilon, \delta \rightarrow 0$ with all the other parameters fixed.

A number of analyses are presented, four of which describe various aspects of the linearised theory and two examine the nature of the far-field nonlinear problem. Using the simplified surface boundary conditions we discuss in turn: the wave motion in the absence of healing: the roble of a second wave speed leading to a wave hierarchy; and the effects of healing. The final linearised problem makes use of the full vapour model, but again the healing terms are ignored. This latter analysis suggests that if the upper boundary of the vapour is sufficiently close to the liquid surface then third sound is suppressed.

The complexity of the equations, particularly when the nonlinear terms are to be examined, is such that the incompressible limit is now taken in the absence of both healing and relaxation. Imposing the physically realistic limiting process $(\varepsilon, \delta \rightarrow 0)$ we show that the only equation valid in the far-field is the Burgers equation. However, we also demonstrate that allowing the other parameters to be functions of $\varepsilon$ (which is not physically realisable in practice) it is easy to derive, for example, the Korteweg-de Vries equation.
\end{abstract}

\footnotetext{
'School of Mathematics, The University, Newcastle upon Tyne, NEI 7RU, U.K.

(1) Copyright Australian Mathematical Society 1984, Serial-fee code 0334-2700/84
} 


\section{Introduction}

The propagation of small amplitude long waves on the surface of superfluid helium (or 'helium II') was first predicted by Atkins [1]. This wave is the analogue of the classical water wave insofar as the body force potential is required for the maintenance of such a motion. In other words we are dealing, in essence with a 'gravity' wave-although here the potential is dominated by van der Waals forces. The surface waves were detected in 1962 by Everitt et al. [7]- and later by Pickar and Atkins [21] - on relatively thick films $(1-10 \mathrm{~cm})$, but our results will be applicable mainly to thin films in the range 5-100 (about 25 atomic layers): see Atkins and Rudnick [2]. The mode of propagation under discussion here is usually referred to as third sound, and there are now known to be quite a few sound speeds associated with superfluid helium. (First sound corresponds to ordinary sound in a classical fluid i.e. a pressure or density wave; second sound is special to the superfluid since the normal and superfluid components (see below) move in opposite directions producing a temperature wave.) In third sound the normal fluid is essentially stationary and only the superfluid oscillates.

The most successful macroscopic theory for helium II is Landau's two-fluid model (Landau [18]; Landau and Lifshitz [19]; Hills and Roberts [9]). The liquid helium below the $\lambda$-point (a second-order phase transition at about $2.2^{\circ} \mathrm{K}$ ) is modelled as a mixture of two component fluids; one moves irrotationally-the superfluid, and the other is a classical (Newtonian) viscous fluid-the normal fluid. It must be emphasised that Landau's approach provides a very convenient model only: it is not to be thought that a quantum fluid like helium II actually is composed of two identifiable fluids. However, on the basis of this model, a considerable body of theory and results has been obtained producing surprisingly good agreement with quite a range of physical phenomena (see, for example, Donnelly [6]; Putterman [22]). Of course, when compared with the problem of surface waves on a classical fluid, it is already clear that the corresponding problem for helium II is vastly more complicated. Unfortunately there are even further difficulties encountered here which have no counterpart in the water-wave problem. Nevertheless, we shall demonstrate that a systematic and useful approach is readily available (which mirrors that used for water waves).

Two novel and curious properties are enjoyed by the superfluid component. The first arises from a description based on the wave function $\Psi$, whence the superfluid density turns out to be proportional to $|\Psi|^{2}$. This means that where the wave function drops to zero then so will the superfluid density: this is the phenomenon of 'healing'. In this work we shall employ the model of healing due to Hills and Roberts [10], which allows the superfluid density to take any assigned value at a boundary of the liquid film. The wave function is certainly zero 
wherever it encounters an infinite potential barrier such as a solid wall, however the free surface requirement is less straightforward. Earlier workers tacitly assume that $\Psi=0$ there; indeed work of Sobaynin [24] and Ginzburg and Sobaynin [8] indicate that almost certainly the superfluid density is zero at such a surface. We shall, where it is necessary, assume that the superfluid density is zero at all boundaries of the liquid. The second novel aspect is the necessity for a constitutive equation describing the relaxation process governing the conversion of normal fluid to superfluid, and vice versa. (In the equilibrium state there is only normal fluid at the $\lambda$-point, and only superfluid at absolute zero.) We shall adopt the relaxation model introduced by Hills and Roberts [10], which in turn is based on that due to Khalatnikov [13]. The details of these various models will become clear when we introduce the equations themselves.

Finally, at the surface of the liquid film, a far more significant but also potentially troublesome condition arises by virtue of the evaporation/condensation between the liquid and its vapour. This produces two effects: first, the surface level can change over and above the usual kinematical contribution; and second, a heat transfer must occur to accommodate the latent heat associated with the exchange. Even with this additional physics, there is still the matter of deciding how to model the vapour. On the one hand we could ignore the vapour altogether and impose appropriate boundary conditions at the surface, solving only for the liquid. Clearly a more satisfactory approach is to model the vapour itself as, say, a classical (Newtonian) compressible gas. We shall examine both choices here and, in the case of the latter, we shall adopt the exact jump conditions developed by Hills and Roberts [11] for helium II with healing and relaxation.

In this paper we shall present the exact equations and boundary conditions, within the framework of the model, that are required for the solution of the surface wave problem. The aim is to analyse these equations by introducing an appropriate (and simple) limiting process which describes the character of the solution under discussion. In this way we avoid making ad hoc approximations (e.g. assuming incompressibility) which are not necessary to the successful completion of the theory. Previous work has tended to start from simplified approximate equations derived by elementary physical arguments rather than precisely defined mathematical operations applied to the exact equations. (For earlier work, which will be referred to in detail later, see in particular Putterman [22]; also Atkins and Rudnick [2], Bergman [3] and [4].) A more systematic approach was adopted in Johnson [15], although rather idealised surface conditions were used in an effort to reduce the complications to a reasonable level. Here we shall-in passing-reproduce this work but a little more emphasis will be given to the solution of the linearised problem coupled with that for the 
vapour. Further, we are able to examine the rôle of the healing terms (which will be ignored in the initial phase of the analysis) and also consider the form of non-linear equations that can arise to describe the wave propagation on long time scales.

The methods we employ here are based on the construction of asymptotic expansions; the procedure is quite straightforward and it lends itself readily to the examination of higher order terms. To this end the equations are non-dimensionalised so as to introduce an amplitude parameter $(\varepsilon)$ and a wavelength parameter $(\delta)$. Since our interest is centred on small amplitude long waves we consider $\varepsilon \rightarrow 0, \delta \rightarrow 0$ keeping all other parameters fixed. It turns out that no other approximations are required to enable the linearised problem to be solved (although the healing terms cause some slight irritation, as we shall see). The philosophy is simply that, for any particular equilibrium state, all the parameters describing the helium II (and its vapour) are given and independent of the amplitude and wave length of the wave to be generated on the surface. Of course once we have obtained propagation speeds, for example, then it may well be expedient to simplify the results by ignoring some of the parameters because they are small (in the strictly numerical-as opposed to the asymptotic limitingsense). However we emphasise that such ad hoc approximations are not an essential ingredient of our approach.

\section{Basic equations and non-dimensionalisation}

The ambient state of the helium is assumed to be a two-dimensional channel of stationary helium II in equilibrium with its vapour. The co-ordinate $x^{\prime}$ (or $x_{1}^{\prime}$ ) measures distance along the channel and $y^{\prime}$ (or $x_{2}^{\prime}$ ) is measured up through the liquid helium from the solid phase (see Figure 1). By virtue of the van der Waals forces of attraction at the wall the helium solidifies to form a layer on the wall; this occurs at a pressure of about $25 \mathrm{~atm}$. for temperatures below about $1.8^{\circ} \mathrm{K}$. We assume that this solid phase is of constant thickness, although the passage of the wave presumably causes a slight fluctuation in the position of the phase boundary. The undisturbed depth of the liquid is $h$ and a typical wavelength of the motion is $\lambda$. (As an alternative a typical frequency could be introduced which is just $\lambda c$, where $c$ is a typical phase speed; see below.) The deviation of the surface away from its equilibrium position is given by $\eta^{\prime}\left(x^{\prime}, t^{\prime}\right)$, where $t^{\prime}$ is a time co-ordinate; the velocity in the liquid is $\mathbf{u}^{\prime} \equiv\left(u^{\prime}, v^{\prime}\right)$ (or $\left(v_{1}^{\prime}, v_{2}^{\prime}\right)$ ). Throughout we shall denote the superfluid and normal components by the subscripts ' $s$ ' and ' $n$ ', respectively. The equations for the liquid phase follow Hills and Roberts [10], and appear in Johnson [15]. 


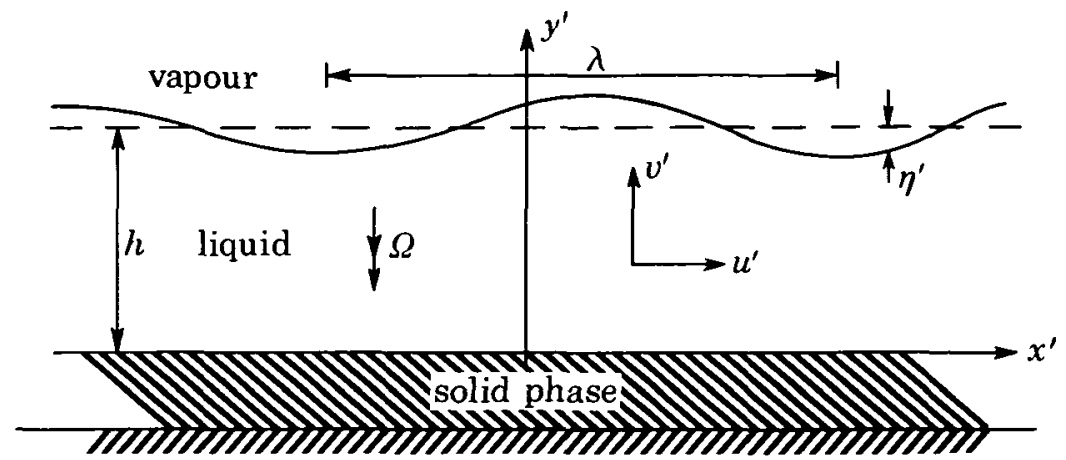

Figure 1. The co-ordinate system and variables.

The conservation of mass, valid at every point interior to the liquid, can be written as

$$
\frac{\partial \rho^{\prime}}{\partial t^{\prime}}+\frac{\partial}{\partial x_{j}^{\prime}}\left(\rho_{n}^{\prime} v_{n j}^{\prime}+\rho_{s}^{\prime} v_{s j}^{\prime}\right)=0,
$$

where $\rho_{n}^{\prime}, \rho_{s}^{\prime}$ are the densities of the components and the density of the mixture is

$$
\rho^{\prime}=\rho_{n}^{\prime}+\rho_{s}^{\prime} \text {. }
$$

The momentum equation for the liquid is

$$
\begin{aligned}
\frac{\partial}{\partial t^{\prime}}\left(\rho_{n}^{\prime} v_{n i}^{\prime}+\rho_{s}^{\prime} v_{s i}^{\prime}\right) & +\frac{\partial}{\partial x_{j}^{\prime}}\left(\rho_{n}^{\prime} v_{n i}^{\prime} v_{n \jmath}^{\prime}+\rho_{s}^{\prime} v_{s i}^{\prime} v_{s \jmath}^{\prime}\right)=-\frac{\partial p^{\prime}}{\partial x_{\imath}^{\prime}}-\rho^{\prime} \frac{\partial \Omega}{\partial x_{\imath}^{\prime}} \\
+ & \frac{\partial}{\partial x_{\jmath}^{\prime}}\left[\mu_{1} \frac{\partial v_{n k}^{\prime}}{\partial x_{k}^{\prime}} \delta_{i j}+\frac{1}{2} \mu_{2}\left(\frac{\partial v_{n i}^{\prime}}{\partial x_{\jmath}^{\prime}}+\frac{\partial v_{n j}^{\prime}}{\partial x_{\imath}^{\prime}}\right)-\frac{\beta^{\prime}}{\rho_{s}^{\prime}} \frac{\partial \rho_{s}^{\prime}}{\partial x_{\imath}^{\prime}} \frac{\partial \rho_{s}^{\prime}}{\partial x_{\jmath}^{\prime}}\right]
\end{aligned}
$$

where $p^{\prime}$ is the pressure, $\Omega$ the body force potential and $\mu_{1}, \mu_{2}$ are the (constant) bulk and shear viscosities, respectively. The healing of the wave function is represented by the term in $\beta^{\prime}$ (a constant), where the coefficient has been taken proportional to $1 / \rho_{s}^{\prime}$ (see Khalatnikov [13]; Hills and Roberts [10]). If we introduce $A\left(\rho^{\prime}, \rho_{s}^{\prime}, T^{\prime}\right)$ - the Helmholtz free energy - where $T^{\prime}$ is the temperature, then the irrotationality of the superfluid is described by

$$
\frac{\partial \varphi_{s}^{\prime}}{\partial t^{\prime}}+\frac{1}{2} v_{s j}^{\prime} v_{s j}^{\prime}+\Omega+A+\rho^{\prime} \frac{\partial A}{\partial \rho^{\prime}}-\frac{1}{2} w_{j}^{\prime} w_{j}^{\prime}-\Lambda m_{s}\left(\rho_{s}^{\prime}\right)^{-1 / 2}=0
$$

where

$$
v_{s i}^{\prime}=\frac{\partial \varphi_{s}^{\prime}}{\partial x_{i}^{\prime}} \quad \text { and } \quad w_{i}^{\prime}=v_{n i}^{\prime}-v_{s i}^{\prime}
$$

The relaxation process appears here by virtue of the term in $\Lambda$ (a constant) with a relaxation coefficient proportional to $\left(\rho_{s}^{\prime}\right)^{-1 / 2}$ (again after Khalatnikov); $m_{s}$ is the 
mass supply function for the superfluid,

$$
m_{s}=\frac{\partial \rho_{s}^{\prime}}{\partial t^{\prime}}+\frac{\partial}{\partial x_{j}^{\prime}}\left(\rho_{s}^{\prime} v_{s j}^{\prime}\right)
$$

The equation of state is

$$
p^{\prime}=\rho^{\prime 2} \frac{\partial A}{\partial \rho^{\prime}}+\rho^{\prime} \rho_{s}^{\prime} \frac{\partial A}{\partial \rho_{s}^{\prime}}-\beta^{\prime} \frac{\partial^{2} \rho_{s}^{\prime}}{\partial x_{j}^{\prime} \partial x_{j}^{\prime}},
$$

and the constitutive equation for superfluid production is

$$
\rho^{\prime} \frac{\partial A}{\partial \rho_{s}^{\prime}}+\frac{1}{2} w_{\jmath}^{\prime} w_{J}^{\prime}+\frac{\beta^{\prime}}{2 \rho_{s}^{\prime 2}}\left[\frac{\partial \rho_{s}^{\prime}}{\partial x_{J}^{\prime}} \frac{\partial \rho_{s}^{\prime}}{\partial x_{j}^{\prime}}-\frac{\partial^{2} \rho_{s}^{\prime}}{\partial x_{j}^{\prime} \partial x_{\jmath}^{\prime}}\right]+\Lambda m_{s}\left(\rho_{s}^{\prime}\right)^{-1 / 2}=0
$$

Finally, remembering that $-\partial A / \partial T^{\prime}=S^{\prime}$ is the entropy, the energy equation becomes

$$
\begin{aligned}
T^{\prime}\left[\frac{\partial}{\partial t^{\prime}}\left(\rho^{\prime} S^{\prime}\right)\right. & \left.+\frac{\partial}{\partial x_{j}^{\prime}}\left(\rho^{\prime} S^{\prime} v_{n \jmath}^{\prime}\right)\right] \\
& =\Lambda\left(\rho_{s}^{\prime}\right)^{-1 / 2} m_{s}^{2}+\frac{\partial}{\partial x_{j}^{\prime}}\left(\kappa^{\prime} \frac{\partial T^{\prime}}{\partial x_{j}^{\prime}}\right)+\mu_{1}\left(\frac{\partial v_{n \jmath}^{\prime}}{\partial x_{j}^{\prime}}\right)^{2}+\mu_{2} \tau_{j k} \tau_{J k},
\end{aligned}
$$

where $\kappa^{\prime}$ is the (constant) thermal conductivity and

$$
\tau_{j k}=\frac{1}{2}\left(\frac{\partial v_{n j}^{\prime}}{\partial x_{k}^{\prime}}+\frac{\partial v_{n k}^{\prime}}{\partial x_{j}^{\prime}}\right)
$$

These equations for the liquid phase, (1), (3), (4), (7), (8) and (9), require boundary conditions on both the bottom (the liquid/solid boundary) at $y^{\prime}=0$ and on the surface at $y^{\prime}=h+\eta^{\prime}$. Since $y^{\prime}=0$ is a solid boundary for the liquid we must have

$$
u_{n}^{\prime}=v_{n}^{\prime}=v_{s}^{\prime}=0 \quad \text { on } y^{\prime}=0,
$$

although it is quite possible for $u_{s}^{\prime} \neq 0$ on $y^{\prime}=0$ since the superfluid is inviscid. Of course there is also a thermal condition on $y^{\prime}=0$ where the temperature or heat transfer is prescribed. It is generally accepted (see Putterman [22]) that the solid phase acts as a perfect insulator, i.e.

$$
\partial T^{\prime} / \partial y^{\prime}=0 \text { on } y^{\prime}=0 .
$$

We now turn to the problem of determining the requirements that are to apply on (and perhaps above) the free surface. It is clear that three types of condition are necessary: kinematic, stress and thermal. These can be obtained by either 
stipulating (almost arbitarily) the conditions that are to pertain on $y^{\prime}=h+\eta^{\prime}$; or the vapour itself can be modelled together with the use of the exact jump conditions valid across an interface. In this work we shall adopt both approaches and compare them (and we note that the former procedure was at the heart of the surface conditions used by Johnson [15]). The first task is to formulate what we might regard as the exact surface conditions. To this end we suppose that the vapour is a compressible gas obeying a Newtonian viscosity law. If we let the vapour be denoted by subscript ' $g$ ' (for gas) then the equations become

$$
\frac{\partial \rho_{g}^{\prime}}{\partial t^{\prime}}+\frac{\partial}{\partial x_{j}^{\prime}}\left(\rho_{g}^{\prime} v_{g J}^{\prime}\right)=0,
$$

(momentum)

$$
\begin{aligned}
\frac{\partial}{\partial t^{\prime}}\left(\rho_{g}^{\prime} v_{g l}^{\prime}\right) & +\frac{\partial}{\partial x_{J}^{\prime}}\left(\rho_{g}^{\prime} v_{g l}^{\prime} v_{g J}^{\prime}\right) \\
= & -\frac{\partial p_{g}^{\prime}}{\partial x_{l}^{\prime}}-\rho_{g}^{\prime} \frac{\partial \Omega}{\partial x_{l}^{\prime}}+\frac{\partial}{\partial x_{\jmath}^{\prime}}\left[\mu_{1 g} \frac{\partial v_{g k}^{\prime}}{\partial x_{k}^{\prime}} \delta_{\imath \jmath}+\mu_{2 g_{g I J}}\right]
\end{aligned}
$$

(state)

$$
p_{g}^{\prime}=\rho_{g}^{\prime 2} \frac{\partial A_{g}}{\partial \rho_{g}^{\prime}} ; \quad A_{g}=A_{g}\left(\rho_{g}^{\prime}, T_{g}^{\prime}\right)
$$

(energy)

$$
\begin{aligned}
T_{g}^{\prime}\left[\frac{\partial}{\partial t^{\prime}}\left(\rho_{g}^{\prime} S_{g}^{\prime}\right)\right. & \left.+\frac{\partial}{\partial x_{\jmath}^{\prime}}\left(\rho_{g}^{\prime} S_{g}^{\prime} v_{g \jmath}^{\prime}\right)\right] \\
& =\frac{\partial}{\partial x_{\jmath}^{\prime}}\left(\kappa_{g}^{\prime} \frac{\partial T_{g}^{\prime}}{\partial x_{\jmath}^{\prime}}\right)+\mu_{1 g}\left(\frac{\partial v_{g \jmath}^{\prime}}{\partial x_{\jmath}^{\prime}}\right)^{2}+\mu_{2 g_{g \jmath k} \tau_{g \jmath k}},
\end{aligned}
$$

where

$$
\tau_{g i j}=\frac{1}{2}\left(\frac{\partial v_{g i}^{\prime}}{\partial x_{\jmath}^{\prime}}+\frac{\partial v_{g \jmath}^{\prime}}{\partial x_{\imath}^{\prime}}\right),
$$

and the notation is exactly as used for equations (1)-(10).

The general interfacial conditions that exist between a liquid and its vapour, and in particular for helium II, have been derived by Hills and Roberts [11]. These jump conditions incorporate the effects of both healing and relaxation and are therefore consistent with our model for the liquid. The conditions are evaluated for our chosen geometry and are merely quoted here. The kinematic condition, which arises from the conservation of mass across the surface, can be written as

$$
\rho_{n}^{\prime}\left(v_{n}^{\prime}-\frac{\partial \eta^{\prime}}{\partial t^{\prime}}-u_{n}^{\prime} \frac{\partial \eta^{\prime}}{\partial x^{\prime}}\right)=\rho_{g}^{\prime}\left(v_{g}^{\prime}-\frac{\partial \eta^{\prime}}{\partial t^{\prime}}-u_{g}^{\prime} \frac{\partial \eta^{\prime}}{\partial x^{\prime}}\right)=J / \cos \theta
$$


where $\tan \theta=\partial \eta^{\prime} / \partial x^{\prime}$ is the slope of the surface. Here, $J$ is the mass flux across $y^{\prime}=h+\eta^{\prime}$ so that $J>0$ corresponds to evaporation from the surface. The continuity of stress at the interface provides an expression for the pressure difference across the free-surface (the normal stress) as well as a relation for the shear stress,

$$
\begin{aligned}
& p^{\prime}-p_{g}^{\prime}+\mu_{i}\left(\frac{\partial u_{g}^{\prime}}{\partial x^{\prime}}+\frac{\partial v_{n}^{\prime}}{\partial y^{\prime}}\right)-\mu_{i}\left(\frac{\partial u_{n}^{\prime}}{\partial x^{\prime}}+\frac{\partial v_{n}^{\prime}}{\partial y^{\prime}}\right) \\
& +\cos ^{2} \theta\left[\mu_{2 g}\left\{\frac{\partial v_{g}^{\prime}}{\partial y^{\prime}}+\left(\frac{\partial \eta^{\prime}}{\partial x^{\prime}}\right)^{2} \frac{\partial u_{g}^{\prime}}{\partial x^{\prime}}-\frac{\partial \eta^{\prime}}{\partial x^{\prime}}\left(\frac{\partial u_{g}^{\prime}}{\partial y^{\prime}}+\frac{\partial v_{g}^{\prime}}{\partial x^{\prime}}\right)\right\}\right. \\
& \left.-\mu_{2}\left\{\frac{\partial v_{n}^{\prime}}{\partial y^{\prime}}+\left(\frac{\partial \eta^{\prime}}{\partial x^{\prime}}\right)^{2} \frac{\partial u_{n}^{\prime}}{\partial x^{\prime}}-\frac{\partial \eta^{\prime}}{\partial x^{\prime}}\left(\frac{\partial u_{n}^{\prime}}{\partial y^{\prime}}+\frac{\partial v_{n}^{\prime}}{\partial x^{\prime}}\right)\right\}\right] \\
& -\left(\frac{1}{\rho^{\prime}}-\frac{1}{\rho_{g}^{\prime}}\right) J^{2}+\frac{\beta^{\prime} \cos ^{2} \theta}{\rho_{s}^{\prime}}\left(\frac{\partial \rho_{s}^{\prime}}{\partial y^{\prime}}-\frac{\partial \eta^{\prime}}{\partial x^{\prime}} \frac{\partial \rho_{s}^{\prime}}{\partial x^{\prime}}\right)^{2}=0,
\end{aligned}
$$

and

$$
\begin{aligned}
\mu_{2}\left[2 \frac{\partial \eta^{\prime}}{\partial x^{\prime}}\left(\frac{\partial u_{n}^{\prime}}{\partial x^{\prime}}-\frac{\partial v_{n}^{\prime}}{\partial y^{\prime}}\right)-\left\{1-\left(\frac{\partial \eta^{\prime}}{\partial x^{\prime}}\right)^{2}\right\}\left(\frac{\partial u_{n}^{\prime}}{\partial y^{\prime}}+\frac{\partial v_{n}^{\prime}}{\partial x^{\prime}}\right)\right] \\
-\mu_{2 g}\left[2 \frac{\partial \eta^{\prime}}{\partial x^{\prime}}\left(\frac{\partial u_{g}^{\prime}}{\partial x^{\prime}}-\frac{\partial v_{g}^{\prime}}{\partial y^{\prime}}\right)-\left\{1-\left(\frac{\partial \eta^{\prime}}{\partial x^{\prime}}\right)^{2}\right\}\left(\frac{\partial u_{g}^{\prime}}{\partial y^{\prime}}+\frac{\partial v_{g}^{\prime}}{\partial x^{\prime}}\right)\right] \\
+\frac{\beta^{\prime}}{\rho_{s}^{\prime}}\left(\frac{\partial \rho_{s}^{\prime}}{\partial x^{\prime}}+\frac{\partial \eta^{\prime}}{\partial x^{\prime}} \frac{\partial \rho_{s}^{\prime}}{\partial y^{\prime}}\right)\left(\frac{\partial \rho_{s}^{\prime}}{\partial y^{\prime}}-\frac{\partial \eta^{\prime}}{\partial x^{\prime}} \frac{\partial \rho_{s}^{\prime}}{\partial x^{\prime}}\right)=0
\end{aligned}
$$

Finally the maintenance of an energy balance across the surface yields

$$
J \Psi=J L+\cos \theta\left[\frac{\partial \eta^{\prime}}{\partial x^{\prime}} \frac{\partial}{\partial x^{\prime}}\left(\kappa_{g}^{\prime} T_{g}^{\prime}-\kappa^{\prime} T^{\prime}\right)-\frac{\partial}{\partial y^{\prime}}\left(\kappa_{g}^{\prime} T_{g}^{\prime}-\kappa^{\prime} T^{\prime}\right)\right] \quad(\geqslant 0),
$$

where $L=T^{\prime}\left(S_{g}^{\prime}-S^{\prime}\right)$ and, in addition, the temperature itself must be continuous so

$$
T^{\prime}=T_{g}^{\prime} \quad \text { on } y^{\prime}=h+\eta^{\prime}
$$

In equation (20) $\Psi$ is a suitable generalisation of the difference in chemical 
potential, and may be written as

$$
\begin{aligned}
\Psi= & A+\frac{p^{\prime}}{\rho^{\prime}}-\left(A_{g}+\frac{p_{g}^{\prime}}{\rho_{g}^{\prime}}\right)+\frac{1}{2}\left(u_{n}^{\prime 2}+v_{n}^{\prime 2}-u_{g}^{\prime 2}-v_{g}^{\prime 2}\right) \\
& +\frac{\rho_{s}^{\prime}}{\rho^{\prime}}\left[\frac{1}{2}\left(u_{n}^{\prime}-u_{s}^{\prime}\right)^{2}+\frac{1}{2}\left(v_{n}^{\prime}-v_{s}^{\prime}\right)^{2}+\Lambda m_{s}\left(\rho_{s}^{\prime}\right)^{-1 / 2}\right]-J \cos \theta\left(\frac{1}{\rho^{\prime}}-\frac{1}{\rho_{g}^{\prime}}\right) \frac{\partial \eta^{\prime}}{\partial t^{\prime}} \\
& +\frac{\mu_{l g}}{\rho_{g}^{\prime}}\left(\frac{\partial u_{g}^{\prime}}{\partial x^{\prime}}+\frac{\partial v_{g}^{\prime}}{\partial y^{\prime}}\right)-\frac{\mu_{1}}{\rho^{\prime}}\left(\frac{\partial u_{n}^{\prime}}{\partial x^{\prime}}+\frac{\partial v_{n}^{\prime}}{\partial y^{\prime}}\right)+\frac{\beta^{\prime}}{2 \rho^{\prime} \rho_{s}^{\prime}} \cos ^{2} \theta\left(\frac{\partial \rho_{s}^{\prime}}{\partial y^{\prime}}-\frac{\partial \eta^{\prime}}{\partial x^{\prime}} \frac{\partial \rho_{s}^{\prime}}{\partial x^{\prime}}\right)^{2} \\
& +\cos ^{2} \theta\left[\frac{\mu_{2 g}}{\rho_{g}^{\prime}}\left\{\frac{\partial v_{g}^{\prime}}{\partial y^{\prime}}+\left(\frac{\partial \eta^{\prime}}{\partial x^{\prime}}\right)^{2} \frac{\partial u_{g}^{\prime}}{\partial x^{\prime}}-\frac{\partial \eta^{\prime}}{\partial x^{\prime}}\left(\frac{\partial u_{g}^{\prime}}{\partial y^{\prime}}+\frac{\partial v_{g}^{\prime}}{\partial x^{\prime}}\right)\right\}\right. \\
& \left.\quad-\frac{\mu_{2}}{\rho^{\prime}}\left\{\frac{\partial v_{n}^{\prime}}{\partial y^{\prime}}+\left(\frac{\partial \eta^{\prime}}{\partial x^{\prime}}\right)^{2} \frac{\partial u_{n}^{\prime}}{\partial x^{\prime}}-\frac{\partial \eta^{\prime}}{\partial x^{\prime}}\left(\frac{\partial u_{n}^{\prime}}{\partial y^{\prime}}+\frac{\partial v_{n}^{\prime}}{\partial x^{\prime}}\right)\right\}\right]
\end{aligned}
$$

To complete the conditions we require a constitutive relation between $J$ and $\Psi$ : Hills and Roberts [11] propose that $J$ be proportional to $\Psi$,

$$
J / \Psi=n^{\prime}\left(p^{\prime}, p_{g}^{\prime}, T^{\prime}\right) \quad(\geqslant 0),
$$

and $n^{\prime}$ can usually be taken as a constant. Of course these surface conditions, (17)-(23), are all to be evaluated on $y^{\prime}=h+\eta^{\prime}$, and (20) has been written in its simplest form by using the requirement that $\rho_{s}^{\prime}=0$ on the free surface. Note that thermodynamic equilibrium between the liquid and its vapour is given by $\Psi=0$, and this must hold when no disturbances are present. The presentation of all the equations describing the two-fluid model of helium II, with healing, relaxation and a vapour model included, is thus complete. The very involved nature of this problem is now manifest, but it is hoped that there is some virtue in presenting here the full set of equations for reference. Clearly, if there is any reasonable possibility of producing a somewhat reduced version of the problem, then there is a case for further investigation. In particular we might expect to be able to write down simpler surface conditions which retain the general character of the problem. This was the philosophy behind the equations given in Johnson [15], which were then regarded as posing an exact problem in its own right. However, this simplified choice of boundary conditions (which we shall also consider here) clearly falls very far short of the formulation given in the equations (13)-(23). Furthermore, it turns out - and it is hardly surprising - that this 'reduced' set of surface conditions can not be obtained from (13)-(23) by any completely systematic limiting procedure.

The alternative model that we adopt is described in detail in Johnson [15], and it follows quite closely the ideas expressed by Atkins and Rudnick [2] and 
Putterman [22]. The helium vapour is assumed to be a gas at constant temperature, $T_{v}^{\prime}$, and constant (vapour) pressure, $p_{v}^{\prime}$. Also the presence of the gas is supposed to produce a zero viscous shearing stress at the surface. These two stress conditions yield

$$
\begin{gathered}
p^{\prime}-\mu_{1}\left(\frac{\partial u_{n}^{\prime}}{\partial x^{\prime}}+\frac{\partial v_{n}^{\prime}}{\partial y^{\prime}}\right) \\
+\cos ^{2} \theta\left[\frac{\beta^{\prime}}{\rho_{s}^{\prime}}\left(\frac{\partial \rho_{s}^{\prime}}{\partial y^{\prime}}-\frac{\partial \eta^{\prime}}{\partial x^{\prime}} \frac{\partial \rho_{s}^{\prime}}{\partial x^{\prime}}\right)^{2}\right. \\
\left.-\mu_{2}\left\{\frac{\partial v_{n}^{\prime}}{\partial y^{\prime}}+\left(\frac{\partial \eta^{\prime}}{\partial x^{\prime}}\right)^{2} \frac{\partial u_{n}^{\prime}}{\partial x^{\prime}}-\frac{\partial \eta^{\prime}}{\partial x^{\prime}}\left(\frac{\partial u_{n}^{\prime}}{\partial y^{\prime}}+\frac{\partial v_{n}^{\prime}}{\partial x^{\prime}}\right)\right\}\right]=p_{v}^{\prime}, \\
\left\{1-\left(\frac{\partial \eta^{\prime}}{\partial x^{\prime}}\right)^{2}\right\}\left(\frac{\partial u_{n}^{\prime}}{\partial y^{\prime}}+\frac{\partial v_{n}^{\prime}}{\partial x^{\prime}}\right)+2 \frac{\partial \eta^{\prime}}{\partial x^{\prime}}\left(\frac{\partial v_{n}^{\prime}}{\partial y^{\prime}}-\frac{\partial u_{n}^{\prime}}{\partial x^{\prime}}\right)=0 .
\end{gathered}
$$

The evaporation/condensation process is assumed to transfer mass and heat in proportion to the difference between the surface temperature and $T_{v}^{\prime}$. If we suppose that there is no superfluid at the free surface then

$$
\begin{aligned}
& \rho^{\prime}\left(v_{n}^{\prime}-\frac{\partial \eta^{\prime}}{\partial t^{\prime}}-u_{n}^{\prime} \frac{\partial \eta^{\prime}}{\partial x^{\prime}}\right)=M^{\prime}\left(T^{\prime}-T_{v}^{\prime}\right) / \cos \theta, \\
& \kappa^{\prime} \cos \theta\left(\frac{\partial T^{\prime}}{\partial y^{\prime}}-\frac{\partial \eta^{\prime}}{\partial x^{\prime}} \frac{\partial T^{\prime}}{\partial x^{\prime}}\right)=-L^{\prime} M^{\prime}\left(T^{\prime}-T_{v}^{\prime}\right) .
\end{aligned}
$$

(These latter two equations show minor alterations when compared with those given in Johnson [15]; here $L^{\prime}$ and $M^{\prime}$ are constants.) Equations (24)-(27), all evaluated on $y^{\prime}=h+\eta^{\prime}$, replace (17)-(20) and we observe that the continuity of temperature, (21), is now redundant since we must not have $T^{\prime} \equiv T_{v}^{\prime}$ on $y^{\prime}=h+$ $\eta^{\prime}$. Comparison of the two sets of surface conditions indicate that, by and large, agreement is obtained if we eliminate the gas terms, e.g. in (18) set $\mu_{1 g}=\mu_{2 g}=0$ and $p_{g}^{\prime}=p_{v}^{\prime}$ but also ignore the term in $J^{2}$. To generate (26) and (27) we model $J$ by $M^{\prime}\left(T^{\prime}-T_{v}^{\prime}\right)$, although we must also use $\Psi=0$ (consistent with the idea of the liquid and vapour being predominantly in equilibrium), ignore (23) and set $\kappa_{g}^{\prime}=0, L=L^{\prime}=$ constant. In fact, if the temperature perturbation is not required, $J$ can be eliminated from the boundary conditions and then the linearised problem solved for the surface wave (as we shall demonstrate): this applies to both pairs (17), (20) and (26), (27).

The final stage in our formulation of the problem is to define an appropriate set of non-dimensional variables. To this end we make use of the length scales $(h, \lambda)$, a typical temperature $T_{0}\left(=T_{v}^{\prime}\right.$ for the simplified model) and a typical density $\rho_{0}$ (for the liquid). The time scale is most conveniently based on $\lambda$ and a 
typical speed $c$. For the vapour we use the density $\rho_{0 g}$ and a speed $c_{g}$ (except for the time variable). The temperature $T_{0}$ (not very close to the $\lambda$-point) is chosen to be the equilibrium temperature in the absence of any disturbances. Now we define

$$
\left.\begin{array}{l}
y^{\prime}=h y, \quad x^{\prime}=\lambda x, \quad t^{\prime}=\lambda t / c, \quad \eta^{\prime}=\varepsilon h \eta, \\
u_{q}^{\prime}=\varepsilon c u_{q}, \quad v_{q}^{\prime}=\varepsilon \delta c v_{q}, \quad \varphi_{s}^{\prime}=\varepsilon \lambda c \varphi_{s}, \quad T^{\prime}=T_{0} T, \\
\rho^{\prime}=\rho_{0} \bar{\rho}, \quad \rho_{q}^{\prime}=\rho_{0} \bar{\rho}_{q}, \quad p^{\prime}=c^{2} \rho_{0} \bar{p}, \quad A=c^{2} a, \quad \Omega=c^{2} \omega, \\
\Psi=c^{2} \psi, \quad J=c \rho_{0} j, \quad n^{\prime}=\rho_{0} \nu / c \quad \text { where } q \equiv n, s,
\end{array}\right\}
$$

and for the vapour

$$
\begin{array}{lll}
u_{g}^{\prime}=\varepsilon c_{g} u_{g}, & v_{g}^{\prime}=\varepsilon \delta c_{g} v_{g}, & T_{g}^{\prime}=T_{0} T_{g}, \\
\rho_{g}^{\prime}=\rho_{0 g} \bar{\rho}_{g}, & p_{g}^{\prime}=c_{g}^{2} \rho_{g} \bar{p}_{g}, & A_{g}=c_{g}^{2} a_{g} .
\end{array}
$$

The various non-dimensional parameters that appear in the equations are given by

$\varepsilon=$ typical wave amplitude $/ h, \quad \delta=h / \lambda, \quad \beta=\beta^{\prime} /(h c)^{2}, \quad \gamma=\Lambda \rho_{0}^{1 / 2} /(h c)$, $\alpha_{i}=\mu_{l} /\left(h c \rho_{0}\right), \quad \alpha_{i g}=\mu_{i g} /\left(h c_{g} \rho_{g}\right) \quad(i=1,2)$, $\kappa=\kappa^{\prime} T_{0} /\left(h \rho_{0} c^{3}\right), \quad \kappa_{g}=\kappa_{g}^{\prime} T_{0} /\left(h \rho_{0 g} c_{g}^{3}\right)$,

and in addition, for the simplified model,

$$
\mu=M^{\prime} T_{0} /\left(c \rho_{0}\right), \quad l=L^{\prime} / c^{2} .
$$

In the above scheme all the physical parameters have been defined with respect to the single length scale $h$ which is prescribed for a given film. The wavelength of third sound is embodied in just one parameter, $\delta$, whence long waves correspond to $\delta \rightarrow 0$ with all other parameters held fixed. The use of $h$ for the gas parameters may appear a little unconventional but, since no limit process is associated with the gas length scale whatever it may be, any convenient choice is admissible. Strictly the same argument can be applied to the choice of $\rho_{0 g}$ and $c_{g}$, but these new scales are retained to facilitate the analysis of the effects of small gas density $\left(\rho_{0 g} / \rho_{0} \ll 1\right)$ in our final equations. Of course, no limit process is implied or employed but two further parameters will therefore appear in the governing equations,

$$
\sigma=\rho_{0_{g}} / \rho_{0} ; \quad \Delta=c_{g} / c .
$$




\section{Equilibrium state and asymptotic expansion}

The problem under consideration here is described by the limit $\varepsilon \rightarrow 0, \delta \rightarrow 0$, i.e. small amplitude long waves, and the relative magnitude of $\varepsilon$ and $\delta$ does not have to be closely specified (although it is not arbitrary in all cases of interest.) As the wave vanishes, so the liquid film and its vapour must approach a state of thermodynamic equilibrium which we shall denote by a circumflex, so $\bar{p}=\hat{p}(y)$, $\bar{p}_{g}=\hat{p}_{g}(y)$, etc. and $T=T_{g}=1$. It should be noted that the equilibrium variables (except temperature) are functions of $y$, and the presence of a wave disturbance will perturb the fluid away from equilibrium. Thus we write

$$
\left.\begin{array}{l}
T=1+\varepsilon \delta \tau, \quad T_{g}=1+\varepsilon \delta \tau_{g}, \quad \bar{p}=\hat{p}+\varepsilon p, \quad \bar{p}_{g}=\hat{p}_{g}+\varepsilon p_{g}, \\
\bar{\rho}=\hat{\rho}+\varepsilon r, \quad \bar{\rho}_{q}=\hat{\rho}_{q}+\varepsilon r_{g} \quad(q=n, s, g),
\end{array}\right\}
$$

which are consistent with the (non-dimensional) equations; the complete set of governing equations, using (28)-(33), is given in the Appendix.

The equations representing mechanical and thermodynamic equilibrium become

$$
\left.\begin{array}{l}
\frac{d \hat{p}}{d y}=-\hat{\rho} \frac{d \omega}{d y}-\beta \frac{d}{d y}\left[\frac{1}{\hat{\rho}_{s}}\left(\frac{d \hat{\rho}_{s}}{d y}\right)^{2}\right], \quad \hat{a}+\hat{\rho} \frac{\partial \hat{a}}{\partial \hat{\rho}}+\omega(y)=0, \\
\hat{p}=\hat{\rho}^{2} \frac{\partial \hat{a}}{\partial \hat{\rho}}+\hat{\rho} \hat{\rho}_{s} \frac{\partial \hat{a}}{\partial \hat{\rho}_{s}}-\beta \frac{d^{2} \hat{\rho}_{s}}{d y^{2}}, \\
\hat{\rho} \frac{\partial \hat{a}}{\partial \hat{\rho}_{s}}-\frac{\beta}{\hat{\rho}_{s}} \frac{d^{2} \hat{\rho}_{s}}{d y^{2}}+\frac{1}{2} \beta\left(\frac{1}{\hat{\rho}_{s}} \frac{d \hat{\rho}_{s}}{d y}\right)^{2}=0,
\end{array}\right\}
$$

for the liquid, where $\hat{a}=a\left(\hat{\rho}, \hat{\rho}_{s}, 1\right)$. (Equations (34) are not independent: for example, the first three imply the fourth upon the elimination of $\hat{\rho}$ and $\omega$.) For the vapour in equilibrium with the helium II we have

$$
\frac{d \hat{\rho}_{g}}{d y}=-\frac{1}{\Delta^{2}} \hat{\rho}_{g} \frac{d \omega}{d y}, \quad \hat{\rho}_{g}=\hat{p}_{g}^{2} \frac{\partial \hat{a}_{g}}{\partial \hat{\rho}_{g}},
$$

where $\hat{a}_{g}=a_{g}\left(\hat{\rho}_{g}, 1\right)$ and at the interface we require 


$$
\left.\begin{array}{l}
\hat{p}-\sigma \Delta^{2} \hat{p}_{g}+\frac{\beta}{\hat{\rho}_{s}}\left(\frac{d \hat{\rho}_{s}}{d y}\right)^{2}=0, \\
\hat{a}+\frac{\hat{p}}{\hat{\rho}}=\Delta^{2}\left(\hat{a}_{g}+\frac{\hat{p}_{g}}{\hat{\rho}_{g}}\right)-\frac{\beta}{2 \hat{\rho} \hat{\rho}_{s}}\left(\frac{d \hat{\rho}_{s}}{d y}\right)^{2}
\end{array}\right\} \quad \text { on } y=1
$$

this latter condition being just $\psi=0$. The equations for the equilibrium state are to be solved with the boundary conditions

$$
\hat{\rho}_{s}=0 \quad \text { on } y=0,1, \quad \hat{p}=\hat{p}_{b} \quad \text { on } y=0,
$$

where $\hat{p}_{b}$ is the (non-dimensional) pressure at which helium II solidifies (at a temperature $T_{0}$ ). It is observed that the rôle of $(36 \mathrm{~b})$ is merely to determine the arbitrary constant in the definition of the Helmholtz free energy for the vapour; the corresponding constant for $\hat{a}$ is fixed by (34b), given $\omega$.

The body force potential, $\omega(y)$, is dominated by the van der Waals force of attraction to the wall which takes the form

$$
\frac{k_{1}}{(y+d)^{3}}\left(1+\frac{y+d}{k_{2}}\right)^{-1},
$$

where $k_{1}, k_{2}$ are constants and $d$ is chosen so that solidification occurs on $y=0$. If $\omega(y)$ is represented solely by (38) then, since $\omega \rightarrow 0$ as $y \rightarrow \infty$, the vapour tends to a constant pressure (and density) far from the surface; this would correspond roughly with the simplified model. However, if the gravitational term were included, then the pressure would decrease with distance above the film (if the film were horizontal) eventually invalidating equations (35). Although it is convenient to consider $y \rightarrow \infty$ in our solution there should be no difficulty in interpreting the results: $y \rightarrow \infty$ is to be taken to mean large on the scale of $h$, which seems perfectly reasonable in the context of the experimental configuration for thin films. We can also note, of course, that any variation with $y$ will be small in practice due to the low density of the helium vapour.

Asymptotic expansions can now be generated by seeking a solution in appropriate powers of $\varepsilon$ and $\delta$, although the precise ordering of these terms cannot be accomplished without specifying a relation between $\varepsilon$ and $\delta$. However the dominant terms that contribute to the perturbation of the equilibrium state can be obtained directly without recourse to a particular $\varepsilon(\delta)$. The equations so formed (in the limit $\varepsilon, \delta \rightarrow 0$ ) are then the basis for the study of the linearised long wave problem. Four different (linearised) problems, of varying complexity, will be discussed in the next paragraph. The simplest starts from the reduced surface conditions and most closely corresponds with the earlier work; other topics include the rôle of the healing terms and of course the use of the 'exact' vapour model. 


\section{Linearised problem}

\section{a. Elementary third sound}

The dominant terms remaining, when $\varepsilon, \delta \rightarrow 0$, are listed below with a reference to the appropriate equation in the Appendix. For these equations to be valid we require, in addition, that $\varepsilon / \delta \rightarrow 0$ in the limit process (see equation $A 7$ ), although we shall also omit the healing terms. This point is discussed in Johnson [15] where it is explained that the significance of the terms in $\beta$ is to produce boundary layers, of $O\left(\beta^{1 / 2}\right)$, on the upper and lower surfaces of the liquid. In other words we are supposing - for the moment-that $\beta$ is very much smaller than $O(1)$, but we shall remove this constraint in a later discussion (see Section $4 \mathrm{c}$ ). The relevant equations are

$$
\frac{\partial r}{\partial t}+\frac{\partial}{\partial x}\left(\hat{\rho}_{n} u_{n}+\hat{\rho}_{s} u_{s}\right)+\frac{\partial}{\partial y}\left(\hat{\rho}_{n} v_{n}+\hat{\rho}_{s} v_{s}\right)=0,
$$

$$
\frac{\partial^{2} u_{n}}{\partial y^{2}}=0, \quad \frac{\partial p}{\partial y}+r \frac{d \omega}{d y}=0
$$

$$
r\left(2 \hat{a}_{\rho}+\hat{\rho} \hat{a}_{\rho \rho}\right)+\frac{\partial \theta_{s}}{\partial t}=0, \quad p=r \hat{\rho}\left(2 \hat{a}_{\rho}+\hat{\rho} \hat{a}_{\rho \rho}\right),
$$

$$
\left(\hat{a}_{T}+\hat{\rho} \hat{a}_{\rho T}\right) \frac{\partial r}{\partial t}+\hat{\rho} \hat{a}_{T} \frac{\partial u_{n}}{\partial x}+\frac{\partial}{\partial y}\left(\hat{\rho} \hat{a}_{T} v_{n}\right)+\kappa \frac{\partial^{2} \tau}{\partial y^{2}}=0,
$$

with

$$
u_{n}=v_{n}=v_{s}=0, \quad \frac{\partial \tau}{\partial y}=0 \quad \text { on } y=0,
$$

and

$$
\left.\begin{array}{lc}
\hat{\rho} v_{n}=\frac{\partial \eta}{\partial t}+\mu \tau, & \frac{\partial u_{n}}{\partial y}=0, \\
\eta \frac{d \hat{p}}{d y}+p=0, & \kappa \frac{\partial \tau}{\partial y}=-\mu l \tau
\end{array}\right\} \quad \text { on } y=1 .
$$

In (4la) we have introduced $\theta_{s}=\theta_{s}(x, t)$ where

$$
\varphi_{s}(x, y, t)=\theta_{s}(x, t)+O\left(\delta^{2}\right),
$$

which is a consequence of the scalings employed on $\left(u_{s}, v_{s}\right)$. Equations (40b), (41) are not independent; it is easily shown, for example, that (40b) follows from the other two with the aid of the equilibrium state equations. The solution of (39)-(44) follows in elementary fashion and yields

$$
u_{n} \equiv 0, \quad p=\int_{y}^{1} r \frac{d \omega}{d y} d y+\hat{\rho}_{1} \omega_{1}^{\prime} \eta=-\hat{\rho} \frac{\partial \theta_{s}}{\partial t}, \quad \frac{\partial \theta_{s}}{\partial t}=-\omega_{1}^{\prime} \eta
$$


and, upon the elimination of $\theta_{s}$ and $\tau_{1}$, we obtain

$$
\begin{array}{r}
{\left[l \hat{\rho}_{1}+\omega_{1}^{\prime} \int_{0}^{1} \frac{\hat{a}_{T}+\hat{\rho} \hat{a}_{\rho T}}{2 \hat{a}_{\rho}+\hat{\rho} \hat{a}_{\rho \rho}} d y+\left(l-\hat{a}_{T 1}\right) \omega_{1}^{\prime} \int_{0}^{1} \frac{d y}{2 \hat{a}_{\rho}+\hat{\rho} \hat{a}_{\rho \rho}}\right] \frac{\partial^{2} \eta}{\partial t^{2}}} \\
-\omega_{1}^{\prime}\left(l-\hat{a}_{T 1}\right)\left(\int_{0}^{1} \hat{\rho}_{s} d y\right) \frac{\partial^{2} \eta}{\partial x^{2}}=0 .
\end{array}
$$

Here we have introduced the additional subscript ' 1 ', denoting evaluation on $y=1$, and the prime is a derivative with respect to $y$. Equation (46) is a wave equation with a (non-dimensional) speed of propagation which is just that labelled $c_{2}$ in Johnson [15]. If we make the further assumption that the liquid is incompressible $\left(2 \hat{a}_{\rho}+\hat{\rho} \hat{a}_{\rho \rho} \rightarrow \infty\right)$ then

$$
c_{2}^{2}=\frac{\omega_{1}^{\prime}}{\hat{\rho}_{1}}\left(1-\frac{\hat{a}_{T 1}}{l}\right) \int_{0}^{1} \hat{\rho}_{s} d y,
$$

which is the expression obtained by Atkins and Rudnick [2] and Putterman [22]. The appearance here of $\hat{\rho}_{1}$, rather than $\hat{\rho}_{n 1}$, is not surprising since $\hat{\rho}_{n 1}=\hat{\rho}_{1}$ i.e. $\hat{\rho}_{s}=0$ at the surface. The equilibrium state is still allowed to retain the healing terms; $\beta$ is neglected only in the perturbation equations. (In fact the presence of superfluid at the free surface gives the same equation, (46), since $\hat{\rho}_{1}$ then arises from $\hat{\rho}_{n 1}+\hat{\rho}_{s 1}$.)

The main features of the flow described by equations (39)-(44) are notably that $u_{n} \equiv 0$, so the normal fluid does not move (as expected) and that the temperature perturbation through the film plays no rôle whatsoever. This latter observation is hardly surprising when it is remembered that the temperature perturbation is $O(\varepsilon \delta)$ and all the other perturbations are $O(\varepsilon)$ : see equations (33). Note, however, that the mass flux and heat transfer at the surface do contribute (even though it turns out that $\hat{a}_{T 1} / l$ is numerically quite small). Finally, the superfluid production equation, (A6), is not required here since this equation uncouples when $\beta$ is small and thus serves only to define $r_{s}$.

\section{b. The wave hierarchy}

The suggestion is made, particularly in Putterman [22], that a second propagation mode (with speed $c_{1}$, say) is available which is also associated with surface wave, i.e. third sound. Actually the dispersion relation developed by Putterman clearly indicates this other mode, although the approximation then used ignores it. To investigate this point further, and to see if this other propagation speed plays any significant rôle, we can derive a single equation embodying both $c_{1}$ and $c_{2}$. The wave equation is then said to describe a wave hierarchy which may, or may not, predict a stable solution (see Whitham [25], [26]). This particular aspect was considered in some detail in Johnson [15], and here we shall give a brief résumé of the analysis. 
Two modes do indeed exist and are linked by a time constant which is proportional to $\mu$, and a balance can be struck between them if $\mu=M \delta$ ( $M$ fixed as $\delta \rightarrow 0$ ). We make this choice and note that it is necessary to write $\tau \rightarrow \tau / \delta$ (so that all perturbations are now $O(\varepsilon)$ : see (33)); as in Section 4a we ignore the healing terms in the perturbation equations. Equations (39), (40), (43), (44a, b, c) are unaltered (if $M$ replaces $\mu$ in (44a)) with

$$
\begin{gathered}
r\left(2 \hat{a}_{\rho}+\hat{\rho} \hat{a}_{\rho \rho}\right)+\tau\left(\hat{a}_{T}+\hat{\rho} \hat{a}_{\rho T}\right)+\frac{\partial \hat{\theta}_{s}}{\partial t}=0, \\
p=r \hat{\rho}\left(2 \hat{a}_{\rho}+\hat{\rho} \hat{a}_{\rho \rho}\right)+\tau \hat{\rho}^{2} \hat{a}_{\rho T}, \\
\left(\hat{a}_{T}+\hat{\rho} \hat{a}_{\rho T}\right) \frac{\partial r}{\partial t}+\hat{\rho} \hat{a}_{T T} \frac{\partial \tau}{\partial t}+\hat{\rho} \hat{a}_{T} \frac{\partial u_{n}}{\partial x}+\frac{\partial}{\partial y}\left(\hat{\rho} \hat{a}_{T} v_{n}\right)+\frac{\kappa}{\delta} \frac{\partial^{2} \tau}{\partial y^{2}}=0, \\
\frac{\kappa}{\delta} \frac{\partial \tau}{\partial y}=-M l \tau \text { on } y=1,
\end{gathered}
$$

replacing (41a, b), (42) and (44d), respectively. These last two equations involve terms in $\delta^{-1}$, although the overall error terms are still $o(1)$. Thus we write

$$
\tau \sim \tau_{0}(x, t)+\delta \tau_{1}(x, y, t)
$$

and it can then be shown that

$$
\tau_{0}=-\left(\eta \omega_{1}^{\prime}+\partial \theta_{s} / \partial t\right) / \hat{a}_{T 1},
$$

and the equation for $\eta(x, t)$ now becomes

$$
\left(\frac{\partial^{2}}{\partial t^{2}}-c_{1}^{2} \frac{\partial^{2}}{\partial x^{2}}\right) \frac{\partial \eta}{\partial t}+m\left(\frac{\partial^{2}}{\partial t^{2}}-c_{2}^{2} \frac{\partial^{2}}{\partial x^{2}}\right) \eta=0 .
$$

The speed $c_{2}$ is exactly that implied by equation (46), $m$ is the appropriate coupling term,

$$
m=\frac{M}{A_{2}}\left(\frac{c_{1}}{c_{2}}\right)^{2} \frac{\omega_{1}^{\prime}}{\hat{a}_{T 1}}\left(l-\hat{a}_{T 1}\right) \quad(>0)
$$

and the speed $c_{1}$ (which turns out to be independent of the latent heat, $l$ ) is given by

$$
c_{1}^{2}=\frac{\omega_{1}^{\prime} \int_{0}^{1} \hat{\rho}_{s} d y}{\hat{\rho}_{1}+\omega_{1}^{\prime} \int_{0}^{1} \hat{\rho}^{2} \alpha_{T} d y-\hat{a}_{T 1} A_{1}^{2} / A_{2}},
$$

where

$$
\begin{aligned}
& A_{1}=\hat{\rho}_{1}+\left(\omega_{1}^{\prime} / \hat{a}_{T 1}\right) \int_{0}^{1} \hat{\rho}\left(\hat{\rho} \hat{a}_{T}+\hat{p}_{T}\right) \alpha_{T} d y \\
& A_{2}=\hat{\rho}_{1} \hat{a}_{T 1}-\left(\omega_{1}^{\prime} \hat{a}_{T 1}\right) \int_{0}^{1}\left[\hat{\rho} \hat{a}_{T T}-\alpha_{T}\left(\hat{\rho} \hat{a}_{T}+\hat{p}_{T}\right)^{2}\right] d y,
\end{aligned}
$$


with

$$
\alpha_{T}=(\hat{\rho} \partial \hat{p} / \partial \hat{\rho})^{-1}=\hat{\rho}^{-2}\left(2 \hat{a}_{\rho}+\hat{\rho} \hat{a}_{\rho \rho}\right)^{-1}, \quad \hat{p}=\hat{p}\left(\hat{\rho}, \hat{\rho}_{s}, T\right) \quad(\equiv \hat{p}(y)) .
$$

The corresponding speed for incompressible flow $\left(\alpha_{T} \rightarrow 0\right)$ is given by

$$
c_{1}^{2}=\frac{1}{\hat{\rho}_{1}}\left(\omega_{1}^{\prime}-\frac{\hat{a}_{T 1}^{2}}{\hat{a}_{T T 1}}\right) \int_{0}^{1} \hat{\rho}_{s} d y,
$$

which again agrees with that obtained by Putterman [22], where it is suggested that (57) is the result of a less careful treatment since $l$ plays no rôle; (47) is regarded as the 'correct' answer. Our presentation makes plain that both speeds are relevant and coupled via the wave hierarchy equation, (54). It is well-known that if $m>0$ and $c_{1}>c_{2}>0$ then the solutions of (54) are stable and describe waves eventually travelling at the speed $c_{2}$ : in this sense (47) is indeed the correct answer. The speeds $c_{1}$ and $c_{2}$ (from (46) and (56)) are plotted in Figure 2 as

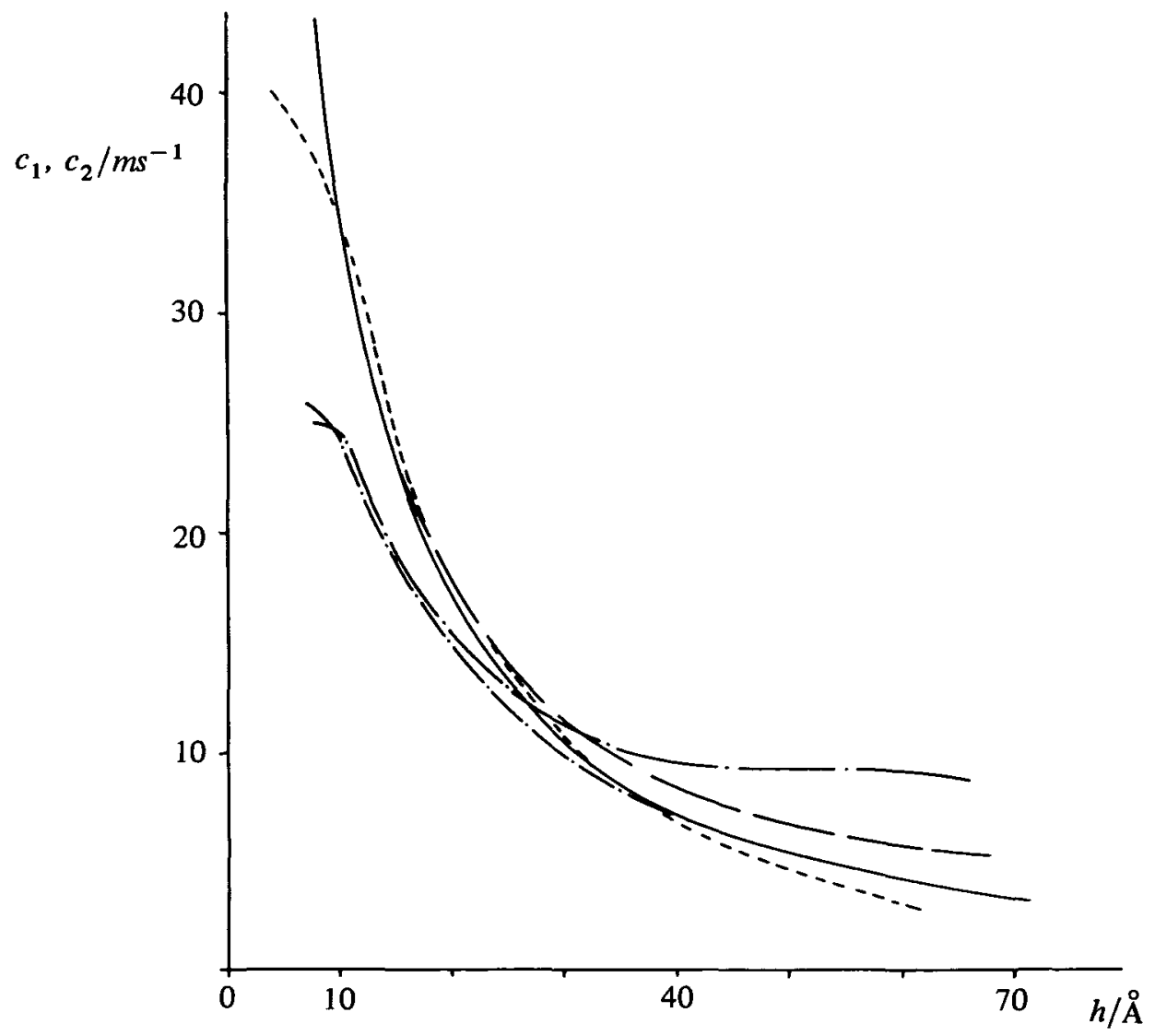

Figure 2. Propagation speeds against film thickness at $T=1.3^{\circ} \mathrm{K}$ : without healing $c_{1}(--), c_{2}(-)$; with healing $c_{1}(\cdot-), c_{2}\left(\cdot-\cdot-\right.$ ). Experimental data curve (Putterman [22]) at $T=1.315^{\circ} \mathrm{K}(-\cdots)$. 
functions of $h$ at a given temperature, and we remark that $c_{2} \rightarrow \infty, c_{1}-c_{2} \rightarrow 0$ as $h \rightarrow 0$. Of course, when $c_{1}$ and $c_{2}$ are close together we can anticipate that the validity of (54) is in some doubt and may well require the inclusion of nonlinear terms. The agreement with the available experimental data is quite reasonable (when compared with $c_{2}$ ) except for very thin films (less than about $20 \AA$ ): see Putterman [22], Johnson [15].

\section{c. The rôle of healing}

One possible explanation for the rather poor agreement with experimental data on very thin layers could be the (arbitrary) disregard of the healing terms in respect of the perturbation equations. Even though the effects of healing were retained in the equilibrium state (and $\beta$ itself turns out to be fairly small-perhaps no larger than about 0.1 ) the possibility that some important new phenomena might have been overlooked must be investigated. This will also serve as a very rigorous test of the continuum two-fluid model of helium II. The aim, therefore, is to examine the perturbation equations (obtained as $\varepsilon, \delta \rightarrow 0$ ) but with $\beta$ fixed. It is immediately clear that the relevant governing equations are much more involved than those discussed in the two previous paragraphs. With this in mind we still limit ourselves to the simplified surface conditions; and to enable estimates for both $c_{1}$ and $c_{2}$ to be obtained we again write $\mu=M \delta, \tau \rightarrow \tau / \delta$ (even though $c_{2}$ may well be the more relevant speed).

Of the equations given earlier (39), (40a), (43), (44a, b) and (51) are unaltered (with $M$ written for $\mu$ in (44a), as before). Now that $\beta=O(1)$ in the limiting process we find that

$$
\begin{gathered}
\frac{\partial p}{\partial y}+r \frac{d \omega}{d y}+\beta \frac{\partial}{\partial y}\left[\frac{2}{\hat{\rho}_{s}} \frac{d \hat{\rho}_{s}}{d y} \frac{\partial r_{s}}{\partial y}-\left(\frac{1}{\hat{\rho}_{s}} \frac{d \hat{\rho}_{s}}{d y}\right)^{2} r_{s}\right]=0 \\
r\left(2 \hat{a}_{\rho}+\hat{\rho} \hat{a}_{\rho \rho}\right)+\tau\left(\hat{a}_{T}+\hat{\rho} \hat{a}_{\rho T}\right)+r_{s}\left(\hat{a}_{\rho_{s}}+\hat{\rho} \hat{a}_{\rho \rho_{s}}\right)+\frac{\partial \theta_{s}}{\partial t}=0 \\
p=r\left(2 \hat{\rho} \hat{a}_{\rho}+\hat{\rho}^{2} \hat{a}_{\rho \rho}+\hat{\rho}_{s} \hat{a}_{\rho_{s}}+\hat{\rho} \hat{\rho}_{s} \hat{a}_{\rho \rho_{s}}\right)+\tau \hat{\rho}\left(\hat{\rho} \hat{a}_{\rho} T+\hat{\rho}_{s} \hat{a}_{\rho_{s} T}\right) \\
+r_{s} \hat{\rho}\left(\hat{\rho} \hat{a}_{\rho \rho_{s}}+\hat{a}_{\rho_{s}}+\hat{\rho}_{s} \hat{a}_{\rho_{s} \rho_{s}}\right)-\beta \frac{\partial^{2} r_{s}}{\partial y^{2}} \\
\left(\hat{a}_{T}+\hat{\rho} \hat{a}_{\rho T}\right) \frac{\partial r}{\partial t}+\hat{\rho}\left(\hat{a}_{T T} \frac{\partial \tau}{\partial t}+\hat{a}_{\rho_{s}} \frac{\partial r_{s}}{\partial t}\right)+\hat{\rho} \hat{a}_{T} \frac{\partial u_{n}}{\partial x}+\frac{\partial}{\partial y}\left(\hat{\rho} \hat{a}_{T} v_{n}\right)+\frac{\kappa}{\delta} \frac{\partial^{2} \tau}{\partial y^{2}}=0 \\
p+\eta\left[\frac{d \hat{p}}{d y}+\beta \frac{d}{d y}\left\{\frac{1}{\hat{\rho}_{s}}\left(\frac{d \hat{\rho}_{s}}{d y}\right)^{2}\right\}\right]+\frac{\beta}{\hat{\rho}_{s}} \frac{d \hat{\rho}_{s}}{d y}\left(2 \frac{\partial r_{s}}{\partial y}-\frac{r_{s}}{\hat{\rho}_{s}} \frac{d \hat{\rho}_{s}}{d y}\right)=0 \quad \text { on } y=1
\end{gathered}
$$


replace (40b), (48), (49), (50) and (44c), respectively. In addition, the superfluid production equation is also required here to define $r_{s}$,

$$
\begin{aligned}
& r\left(\hat{a}_{\rho_{s}}+\hat{\rho} \hat{a}_{\rho \rho_{s}}\right)+r_{s} \hat{\rho} \hat{a}_{\rho_{s} \rho_{s}}+\tau \hat{\rho} \hat{a}_{\rho_{s} T} \\
& -\frac{\beta}{\hat{\rho}_{s}^{3}}\left[\hat{\rho}_{s}^{2} \frac{\partial^{2} r_{s}}{\partial y^{2}}-\hat{\rho}_{s} \frac{d^{2} \hat{\rho}_{s}}{d y^{2}} r_{s}-\hat{\rho}_{s} \frac{d \hat{\rho}_{s}}{d y} \frac{\partial r_{s}}{\partial y}+\left(\frac{d \hat{\rho}_{s}}{d y}\right)^{2} r_{s}\right]=0,
\end{aligned}
$$

and since $\rho_{s}=0$ on $y=0,1+\varepsilon \eta$ we seek the solution for which $r_{s}=0$ on $y=0,1$. (This follows since $\hat{\rho}_{s} \rightarrow 0$ quadratically as $y \rightarrow 0,1$.) However, $\rho_{s} \rightarrow 0$ does not imply that the relaxation terms become unbounded near $y=0,1$ (see Johnson [16]): these terms remain uniformly small as $\varepsilon, \delta \rightarrow 0$.

It is clear that the real difficulty in this problem stems from the behaviour of the variables through the layer as functions of $y$. Given a suitable form for $a\left(\rho, \rho_{s}, T\right)$ (which is best constructed from the available data for helium II) the equilibrium state must be determined and this can be used to find all the necessary information for the solution of the perturbation equations. This procedure, which is mainly numerical, is adopted in Johnson [17], and only the barest outline will be described here. In fact the underlying structure of the problem is no different from that discussed in Section $4 \mathrm{~b}$. We find that

$$
u_{n} \equiv 0, \quad \tau \sim \tau_{0}(x, t)+\delta \tau_{1}(x, y, t)
$$

and then

$$
\int_{0}^{1}\left[\left(\hat{a}_{T}+\hat{\rho} \hat{a}_{\rho T}\right) \frac{\partial r}{\partial t}+\hat{\rho}\left(\hat{a}_{T T} \frac{\partial \tau_{0}}{\partial t}+\hat{a}_{\rho s} \frac{\partial r_{s}}{\partial t}\right)\right] d y+\hat{a}_{T 1}\left(\frac{\partial \eta}{\partial t}+M \tau_{0}\right)=M l \tau_{0}
$$

with, for example,

$$
\eta \frac{d \omega}{d y}+\frac{\partial \theta_{s}}{\partial t}+\hat{a}_{T} \tau_{0}=\frac{\beta}{\hat{\rho} \hat{\rho}_{s}}\left(\frac{d \hat{\rho}_{s}}{d y} \frac{\partial r_{s}}{\partial y}-\frac{d^{2} \hat{\rho}_{s}}{d y^{2}} r_{s}\right) \quad \text { on } y=1 .
$$

The equation for $r_{s}$ can be written as

$$
\begin{aligned}
\beta \frac{\partial}{\partial y}\left[\frac { 1 } { \hat { \rho } _ { s } } \left(\frac{d^{2} \hat{\rho}_{s}}{d y^{2}} r_{s}\right.\right. & \left.\left.-\frac{d \hat{\rho}_{s}}{d y} \frac{\partial r_{s}}{\partial y}\right)\right]+\hat{\rho}^{2} \alpha_{T}\left(\hat{a}_{\rho_{s}}+\hat{\rho} \hat{a}_{\rho \rho_{s}}\right) \frac{d \omega}{d y} r_{s} \\
& +\left(\frac{d \hat{p}}{d y}+\hat{\rho}^{2} \alpha_{T} \frac{d \omega}{d y}\right) \frac{\partial \theta_{s}}{\partial t}+\left[\frac{d}{d y}\left(\hat{\rho} \hat{a}_{T}\right)+\hat{\rho}^{2} \alpha_{T}\left(\hat{a}_{T}+\hat{\rho} \hat{a}_{\rho T}\right)\right] \tau_{0}=0,
\end{aligned}
$$

so that we can express the solution as

$$
r_{s}=\tau_{0} R(y)+\frac{\partial \theta_{s}}{\partial t} Q(y)
$$


where $R(y), Q(y)$ satisfy second order non-homogeneous ordinary differential equations. Since the continuity equation yields

$$
\int_{0}^{1}\left(\frac{\partial r}{\partial t}+\hat{\rho}_{s} \frac{\partial^{2} \theta_{s}}{\partial x^{2}}\right) d y+\frac{\partial \eta}{\partial t}+M \tau_{0}=0
$$

we can eliminate $\theta_{s}$ and $\tau_{0}$ between (64), (65) and (66) (using (59) for $r$ ) to give (54) again

$$
\left(\frac{\partial^{2}}{\partial t^{2}}-c_{1}^{2} \frac{\partial^{2}}{\partial x^{2}}\right) \frac{\partial \eta}{\partial t}+m\left(\frac{\partial^{2}}{\partial t^{2}}-c_{2}^{2} \frac{\partial^{2}}{\partial x^{2}}\right) \eta=0 .
$$

The speeds $c_{1}, c_{2}$ and coupling constants $m$ are now, however, rather more complicated. For example, we find that

$$
c_{2}^{2}=\frac{\omega_{1}^{\prime}\left(l-\hat{a}_{T 1}\right) \int_{0}^{\prime} \hat{\rho}_{s} d y}{\omega_{1}^{\prime}\left(I_{1}+\left(l-\hat{a}_{T 1}\right) I_{2}\right)+l \hat{\rho}_{1}\left(1-\beta Q^{\prime \prime}(1) / \hat{\rho}_{1}\right)},
$$

where

$$
\begin{aligned}
& I_{1}=\int_{0}^{1}\left[\hat{\rho} \hat{\alpha}_{T}\left(\hat{\rho} \hat{a}_{T}+\hat{p}_{T}\right)+Q\left\{\chi\left(\hat{a}_{T}+\hat{\rho} \hat{a}_{\rho T}\right)-\hat{\rho} \hat{a}_{\rho_{s} T}\right\}\right] d y, \\
& I_{2}=\int_{0}^{1}\left(\hat{\rho}^{2} \alpha_{T}+Q \chi\right) d y ; \quad \chi=\hat{\rho}^{2} \alpha_{T}\left(\hat{a}_{\rho_{s}}+\hat{\rho} \hat{a}_{\rho \rho_{s}}\right),
\end{aligned}
$$

which agrees with our previous expression for $c_{2}$ if the healing terms are ignored i.e. $Q \equiv 0$. Two important observations can be made about (68): first, the limit to incompressibility $\left(\alpha_{T} \rightarrow 0\right)$ retains the term $Q^{\prime \prime}(1)$ arising from the superfluid perturbation, and second we see that $c_{2}$ depends only on $Q$ (not on $R$ as well; see (66)). Thus the assumption of an incompressible liquid does not seem to automatically rule out any connection with the phenomenon of healing. Since $c_{2}$ is independent of $R$, this speed is not associated with the temperature perturbation $\left(\tau_{0}\right)$ through the layer which, of course, we already know. If we revert to $\mu$ (rather than $M$ ) then $\tau_{0}$ is absent from the analysis altogether and a wave equation involving $c_{2}$ only is obtained (see Section 4a). The speed $c_{1}$, and $m$, are even more complicated than $c_{2}$ and they do depend on both $Q$ and $R$.

The main aim of such an analysis as this is to produce predictions for the speed of propagation of the surface wave, which means essentially $c_{2}$. However, because the equilibrium state must also be determined in some detail we obtain in addition, for example, the superfluid density distribution across the layer. Apart from showing the way in which the zero boundary conditions are accommodated - and giving estimates for the boundary layer (healing) thickness-the 'onset' phenomenon can also be investigated. This is the observation that below a critical thickness (at a given temperature) no wave propagates: the accepted interpretation is that $\hat{\rho}_{s}$ has dropped to zero right across the layer (see Chester and Yang, [5]). Surprisingly, just this result is found from the numerical analysis even though 
the thickness is as little as about $5 \AA$ (between one and two atomic layers): a remarkable consequence of a continuum theory. Full details of all these calculations are to be found in Johnson [17], but a few results are given in Figures 2 and 3. Three fairly typical superfluid density profiles are shown in Figure 3, and the variation of $c_{1}$ and $c_{2}$ with depth appears in Figure 2. The quite dramatic effects of healing-predominantly the reduction in the value of $\int_{0}^{1} \hat{\rho}_{s} d y$-are evident here where the theoretical curves start to turn over as $h \rightarrow 0$. (Of course, at the onset thickness, then $c_{1}=c_{2}=0$.) On balance, the inclusion of the healing terms would seem to produce rather disappointing results: the effect of these terms is, if anything, too potent.

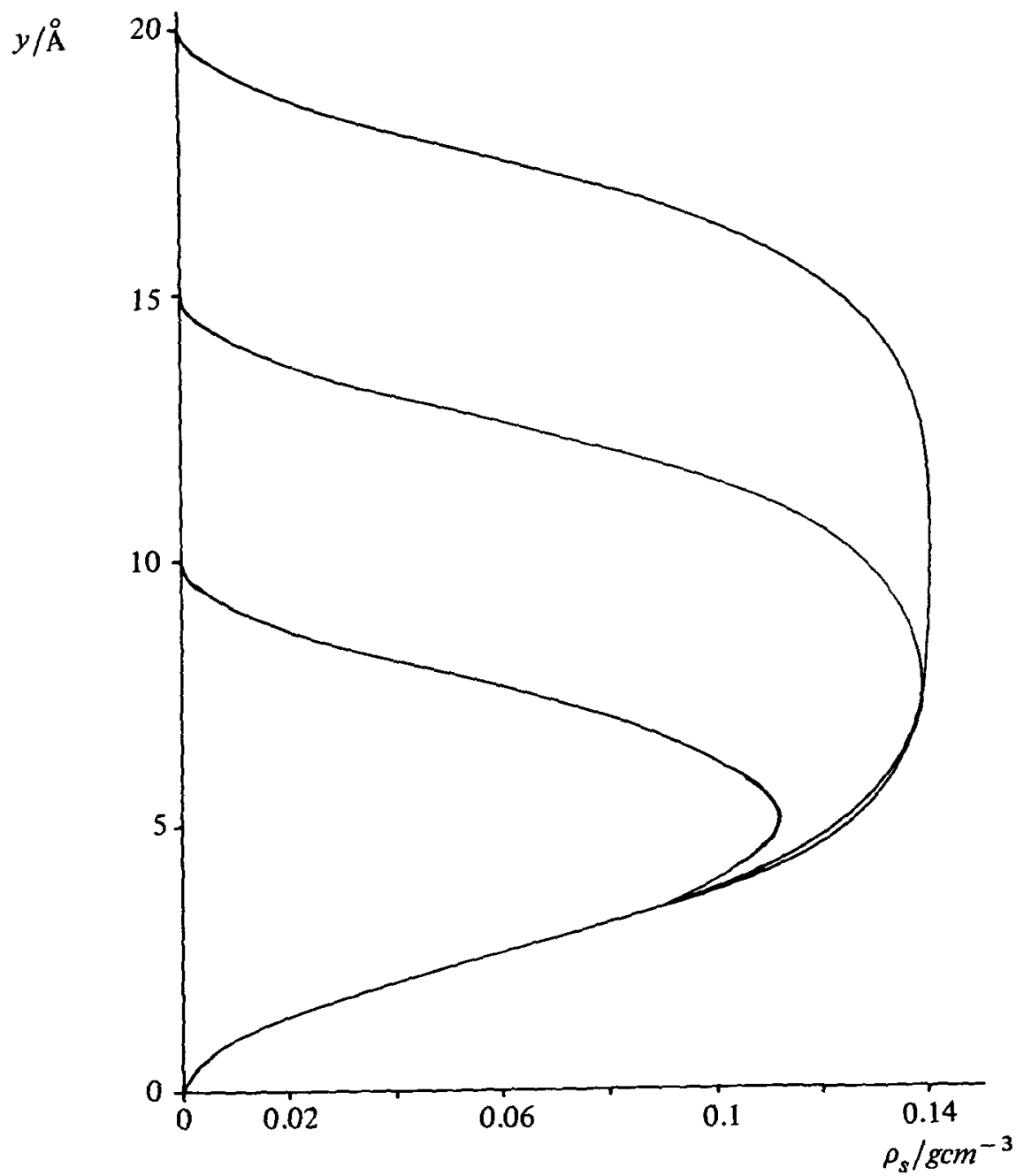

Figure 3. The equilibrium superfluid density at $T=1.3^{\circ} \mathrm{K}$ for three film thicknesses: $10,15,20 \dot{A}$. 


\section{d. The vapour model}

In the three previous analyses of the problem of third sound we have used exclusively the simplified surface boundary conditions (24)-(27). It is, however, to be expected that a model of the vapour coupled with accurate conditions on $y=1+\varepsilon \eta$ should more closely conform with the experimental configuration. To best emphasize the rôle of the vapour it is convenient to restrict the calculation so that it corresponds to that discussed in Section 4a. Thus we shall ignore the healing terms and retain $\mu=O(1)$ as $\varepsilon, \delta \rightarrow 0$ : this latter constraint implies that we expect to obtain $c_{2}$ propagation, but not $c_{1}$. Since we aim to give a little more detail here we shall quote all the relevant perturbation equations (although some of them have appeared earlier). The equilibrium state is given by the complete set (34)-(36), and then for the liquid (see (39)-(42))

$$
\left.\begin{array}{c}
\frac{\partial r}{\partial t}+\frac{\partial}{\partial x}\left(\hat{\rho}_{n} u_{n}+\hat{\rho}_{s} u_{s}\right)+\frac{\partial}{\partial y}\left(\hat{\rho}_{n} v_{n}+\hat{\rho}_{s} v_{s}\right)=0, \\
\frac{\partial^{2} u_{n}}{\partial y^{2}}=0, \quad \frac{\partial p}{\partial y}+r \frac{d \omega}{d y}=0, \\
r\left(2 \hat{a}_{\rho}+\hat{\rho} \hat{a}_{\rho \rho}\right)+\frac{\partial \theta_{s}}{\partial t}=0, \quad p=r \hat{\rho}\left(2 \hat{a}_{\rho}+\hat{\rho} \hat{a}_{\rho \rho}\right), \\
\left(\hat{a}_{T}+\hat{\rho} \hat{a}_{\rho T}\right) \frac{\partial r}{\partial t}+\hat{\rho} \hat{a}_{T} \frac{\partial u_{n}}{\partial x}+\frac{\partial}{\partial y}\left(\hat{\rho} \hat{a}_{T} v_{n}\right)+\kappa \frac{\partial^{2} \tau}{\partial y^{2}}=0 .
\end{array}\right\}
$$

The vapour, modelled as a Newtonian compressible gas, is described to leading order by

$$
\begin{aligned}
& (\mathrm{A} 10,11) \quad \frac{1}{\Delta} \frac{\partial r_{g}}{\partial t}+\frac{\partial}{\partial x}\left(\hat{\rho}_{g} u_{g}\right)+\frac{\partial}{\partial y}\left(\hat{\rho}_{g} v_{g}\right)=0, \quad \frac{\partial^{2} u_{g}}{\partial y^{2}}=0, \\
& (\mathrm{~A} 12,13) \quad \frac{\partial p_{g}}{\partial y}+\frac{r_{g}}{\Delta^{2}} \frac{d \omega}{d y}=0, \quad p_{g}=r_{g} \hat{\rho}_{g}\left(2 \hat{a}_{g \rho}+\hat{\rho}_{g} \hat{a}_{g \rho \rho}\right), \\
& \text { (A14) } \frac{1}{\Delta}\left(\hat{a}_{g T}+\hat{\rho}_{g} \hat{a}_{g \rho}\right) \frac{\partial r_{g}}{\partial t}+\hat{\rho}_{g} \hat{a}_{g T} \frac{\partial u_{g}}{\partial x}+\frac{\partial}{\partial y}\left(\hat{\rho}_{g} \hat{a}_{g T} v_{g}\right)+\kappa_{g} \frac{\partial^{2} \tau_{g}}{\partial y^{2}}=0 .
\end{aligned}
$$

The boundary conditions at the bottom of the liquid layer are the standard ones $(A 8,9)$, but at the liquid/vapour interface we now have

$$
(\mathrm{A} 15,18) \quad \begin{aligned}
\hat{\rho}_{1}\left(v_{n}-\frac{\partial \eta}{\partial t}\right) & =\Delta \sigma \hat{\rho}_{g 1}\left(v_{g}-\frac{1}{\Delta} \frac{\partial \eta}{\partial t}\right) \\
& =\left(\kappa \frac{\partial \tau}{\partial y}-\Delta^{3} \sigma \kappa_{g} \frac{\partial \tau_{g}}{\partial y}\right) /\left(\Delta^{2} \hat{a}_{g T 1}-\hat{a}_{T 1}\right),
\end{aligned}
$$


obtained by eliminating $j / \cos \theta$ and using the fact that $\psi=O(\varepsilon \delta)$, which itself implies that

$$
\frac{p}{\hat{\rho}_{1}}=\Delta^{2} \frac{p_{g}}{\hat{\rho}_{g 1}} .
$$

(Note that (A20) is redundant to this order.) Also

$$
(\mathrm{A} 16,17) \quad p+\eta \hat{p}^{\prime}=\sigma \Delta^{2}\left(p_{g}+\eta \hat{p}_{g}^{\prime}\right), \quad \alpha_{2} \frac{\partial u_{n}}{\partial y}=\alpha_{2 g} \sigma \Delta^{2} \frac{\partial u_{g}}{\partial y},
$$

being the appropriate approximations to the stress conditions; equations (73)-(75) are evaluated on $y=1$. The boundary conditions to be applied at the outer edge of the vapour (i.e. far from the liquid surface) will be discussed in some detail shortly.

Two points are worthy of note in our particular formulation of the interface conditions. One we raised earlier and concerns the arbitrariness in the modelling of $j$ : the required surface condition can be taken as (73), which agrees with our previous results if we ignore the gas terms (and delete the mass flux in the gas). In other words, for example, we could eliminate $\mu \tau$ between (44a) and (44d) provided we did not specifically require the temperature perturbation at the surface. The second confirms the condition $\Psi=0$ in the simplified analysis for, in the above, we have shown that a consequence of the scalings is just $\psi=O(\varepsilon \delta)$. Thus the 'reduced' boundary conditions correlate quite well with our limit of the exact conditions. Nevertheless some difficulties still remain if we attempt to eliminate the gas and thereby recover precisely (24)-(27), although much of the detail certainly is accurate.

If $u_{g} \rightarrow 0$ as $y \rightarrow \infty$ then the solution of (69b), (70a), (75b) with $u_{n}=0$ on $y=0$ is simply

$$
u_{n}=u_{g} \equiv 0 \text {, }
$$

so that, as before, the normal fluid is stationary and the vapour is 'locked' to it via the shear stress at the surface. We then obtain for the liquid

$$
\hat{\rho}_{1} v_{n 1}+\int_{0}^{1}\left(\frac{\partial r}{\partial t}+\hat{\rho}_{s} \frac{\partial^{2} \theta_{s}}{\partial x^{2}}\right) d y=0,
$$

with $r=\hat{\rho}^{2} \alpha_{T} \omega_{1}^{\prime} \eta$ and $\partial \theta_{s} / \partial t=-\omega_{1}^{\prime} \eta$, and the energy equation yields

$$
\hat{\rho}_{1} \hat{a}_{T 1} v_{n 1}+\kappa\left(\frac{\partial \tau}{\partial y}\right)_{1}+\int_{0}^{1}\left(\hat{a}_{T}+\hat{\rho} \hat{a}_{\rho T}\right) \frac{\partial r}{\partial t} d y=0 .
$$

Here we have used

$$
p=\hat{\rho}_{1} \omega_{1}^{\prime} \eta \text { on } y=1 \text {, }
$$


which follows from (74), (75a) and the equilibrium state, as does the corresponding result for the gas

$$
p_{g}=\frac{1}{\Delta^{2}} \hat{\rho}_{g 1} \omega_{1}^{\prime} \eta \quad \text { on } y=1
$$

The gas density is then given by

$$
r_{g}=\frac{\alpha_{g T}}{\Delta^{2}} \hat{\rho}_{g} \hat{\rho}_{g 1} \omega_{1}^{\prime} \eta \exp \left[-\Delta^{-2} \int_{1}^{y} \omega^{\prime} \hat{\rho}_{g} \alpha_{g T} d y\right]
$$

where $\hat{\rho}_{g}^{2} \alpha_{g T}=\left(2 \hat{a}_{g \rho}+\hat{\rho}_{g} \hat{a}_{g \rho \rho}\right)^{-1}$, and for mass conservation

$$
\hat{\rho}_{g 1} v_{g 1}-\frac{1}{\Delta} \int_{1}^{\infty} \frac{\partial r_{g}}{\partial t} d y=m_{g}
$$

if $m_{g}$ is the mass flux at infinity (outwards). It might be expected that $m_{g}$ should be zero but we shall demonstrate that, for third sound to exist, this cannot be the case. Similarly from the energy equation (72) we obtain

$$
\hat{\rho}_{g 1} \hat{a}_{g T 1} v_{g 1}+\kappa_{g}\left(\frac{\partial \tau_{g}}{\partial y}\right)_{1}-\frac{1}{\Delta} \int_{1}^{\infty}\left(\hat{a}_{g T}+\hat{\rho}_{g} \hat{a}_{g \rho}\right) \frac{\partial r_{g}}{\partial t} d y=m_{g} \hat{a}_{g T \infty}
$$

where we have accounted for the (inward) flow of entropy at infinity, and assumed that $\partial \tau_{g} / \partial y \rightarrow 0$ as $y \rightarrow \infty$. The necessity for the inclusion of the terms in $m_{g}$ is quite easily explained when we look a little more closely at the process of evaporation/condensation. A trough of the wave is (relatively) hotter and causes evaporation there directly away from the surface, while at a peak the reverse pertains. At distances comparable with $h$ (and $y$ is scaled on $h$ ) this process is limited to the $y$-direction. Of course, on larger distance scales there is presumably a continuous transfer of material from troughs to peaks (see Figure 4), so maintaining the overall conservation laws. The problem for the outer region of the vapour can be treated separately since to leading order this is uncoupled from our solution (provided there is no heat transfer from the outer vapour). However, if we use the boundary conditions

$$
v_{g}=0, \quad \partial \tau_{g} / \partial y=0
$$

at finite $y(>1)$, or $y \rightarrow \infty$, then $m_{g}=0$ and it is easily shown that $\partial \eta / \partial x=$ $\partial \eta / \partial t=0$. Thus if the upper boundary is brought too close to the surface of the helium II it suggests that the wave will be entirely suppressed.

To complete the analysis we eliminate $m_{g}$ between (78) and (79), express $v_{g 1}$ in terms of $v_{n 1}$ (from (73)), substitute for the heat transfer terms in (73) and finally 
use (76) for $v_{n !}$. This yields the wave equation for $\eta(x, t)$

$$
\begin{aligned}
& {\left[l_{\infty} \hat{\rho}_{1}+\sigma \hat{\rho}_{g 1}\left(l_{1}-l_{\infty}\right)+\omega_{1}^{\prime} \int_{0}^{1}\left(\hat{a}_{T}+\hat{\rho} \hat{a}_{\rho T}\right) \hat{\rho}^{2} \alpha_{T} d y\right.} \\
& \left.+\left(l_{\infty}-\hat{a}_{T 1}\right) \omega_{1}^{\prime} \int_{0}^{1} \hat{\rho}^{2} \alpha_{T} d y+\sigma \omega_{1}^{\prime} \int_{1}^{\infty}\left(\hat{a}_{g T}+\hat{\rho}_{g} \hat{a}_{g \rho T}-\hat{a}_{g T \infty}\right) f_{g} d y\right] \frac{\partial^{2} \eta}{\partial t^{2}} \\
& -\omega_{1}^{\prime}\left(l_{\infty}-a_{T 1}\right)\left(\int_{0}^{1} \hat{\rho}_{s} d y\right) \frac{\partial^{2} \eta}{\partial x^{2}}=0,
\end{aligned}
$$

where $l_{l}=\hat{a}_{T l}-\Delta^{2} \hat{a}_{g T l}, \quad l_{\infty}=\hat{a}_{T l}-\Delta^{2} \hat{a}_{g T \infty}$ and we have written $r_{g}=$ $\left(\omega_{1}^{\prime} \eta / \Delta^{2}\right) f_{g}(y)$. This is to be compared with equation (46). The limit which now 'removes' the gas is simply $\sigma \rightarrow 0$, keeping all the other parameters fixed. If we interpret $l_{\infty}$ as $l$ (see (31)) then the agreement with (46) is achieved. Of course, as it stands, (80) is valid for arbitrary density ratios $(\sigma)$ and the additional simplifications here seem reasonable since $\sigma$ is about 0.0014 for helium II and its vapour. However, the limit $\sigma \rightarrow 0$ presumably implies some appropriate limit in $\Delta$ : from (36a) the natural choice is $\sigma \Delta^{2}=1$ if we bear in mind that the pressures are equal (in the absence of healing). With this choice, the limit $\sigma \rightarrow 0$ gives a wave speed $c_{2}$ with

$$
c_{2}^{2}=\frac{\omega_{1}^{\prime} \int_{0}^{1} \hat{\rho}_{s} d y}{\hat{\rho}_{1}+\omega_{1}^{\prime} \int_{0}^{1} \hat{\rho}^{2} \alpha_{T} d y},
$$

which in turn is just

$$
c_{2}^{2}=\left(\omega_{1}^{\prime} / \hat{\rho}_{1}\right) \int_{0}^{1} \hat{\rho}_{s} d y
$$

if the liquid is assumed incompressible $\left(\alpha_{T} \rightarrow 0\right)$. This latter expression can be recovered from (74) when the condition $\left|\hat{a}_{T 1} / l\right| \ll 1$ is incorporated: our limit implies that $\hat{a}_{g T_{\infty}} / l_{\infty}=O(\sigma)$.

Although the outer regions of the vapour have not been analysed, we can make one or two observations about this problem. The flow here should accommodate appropriate boundary conditions very far from the surface e.g. $u_{g} \rightarrow 0, v_{g} \rightarrow 0$, $\partial \tau_{g} / \partial y \rightarrow 0$, and permit the mass and entropy re-circulation (appearing in (78), (79)) as the surface is approached from above. The actual mathematical character of this problem is quite involved mainly because it depends critically on the choice of $\omega(y)$. For example, if $\omega(y)$ incorporates only the van der Waals term then $p_{g}$ and $r_{g}$ become independent of $y$ as $y \rightarrow \infty$ (since $\omega(y) \rightarrow 0$ ). On the other hand if $\omega(y)$ is modelled to include gravity then $p_{g}, r_{g} \rightarrow 0$ as $y \rightarrow \infty$, and this approach is exponential. Without being specific about $\omega$, the scalings associated with this outer region therefore cannot be determined and so neither can we find the corresponding governing equations to leading order. Fortunately, as we have demonstrated, the vapour does not affect the surface wave provided the gas does 
not conduct heat to/from the neighbourhood of the interface. For a small density ratio $(\sigma \rightarrow 0)$ the presence of the gas is still relevant in that $l_{\infty}\left(i . e . \hat{a}_{g T_{\infty}}\right)$ rather than $l_{1}$ appears in the final expression for the wave speed (assuming $l_{\infty}$ is fixed in the limit so as to produce essentially (46)).

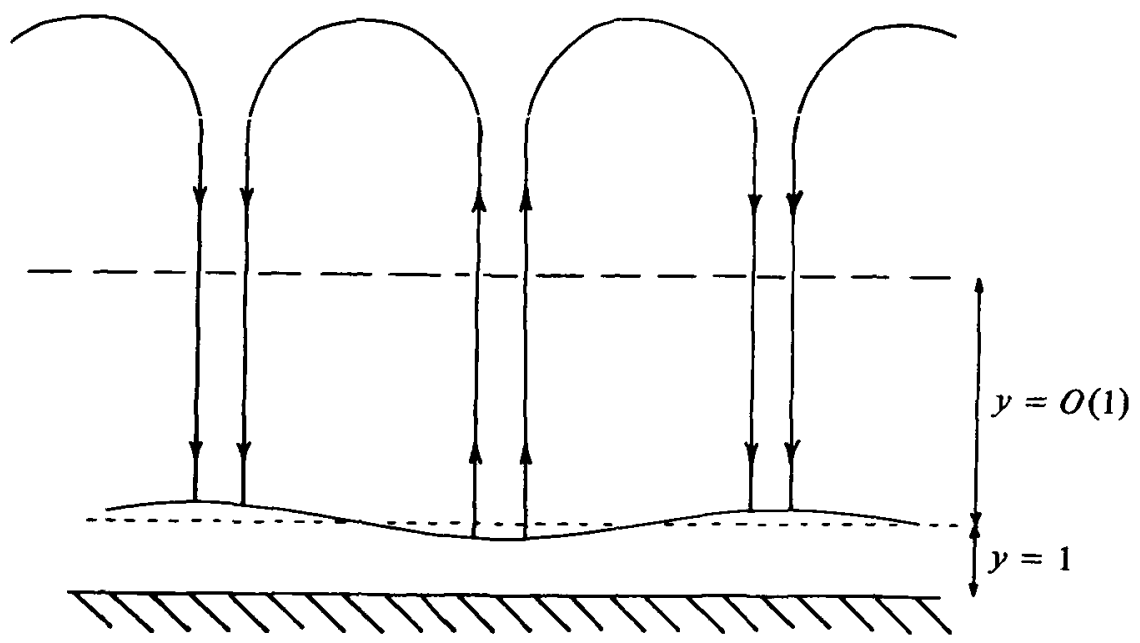

Figure 4. A schematic representation of the evaporation, condensation and re-circulation in third sound.

\section{Nonlinear theory}

\section{a. Burgers equation}

The four discussions in Section 4 have been restricted to various forms of the linearised problem but the full non-dimensional equations enable higher order terms to be obtained (at least in principle). These terms, regarded as corrections to the linear theory, are not of any great interest, however they do indicate the character of the nonlinear problem. In wave propagation it is more usual-and far more convenient-to construct directly the appropriate far-field equation which embodies the nonlinearity as well as (presumably) dispersive and/or dissipative terms. The problem of third sound is no exception, although the complexity of the equations and the plethora of parameters makes the task far from straightforward. Nevertheless, if we restrict the limit process to just $\varepsilon, \delta \rightarrow 0$ (which is consistent with our philosophy) and choose to discuss a suitably simplified set of equations the analysis is quite manageable. In order to examine the general character of the nonlinear problem we shall confine ourselves to a model of liquid helium which is both incompressible and without healing and relaxation. Thus in equations (A1)-(A9) we set $\beta=\gamma=0, \hat{\rho}=$ constant ( $\hat{r} \equiv 0)$ and eliminate $\hat{\rho} a_{\rho}$ between (A4) and (A5) whence (AS) is then redundant. The simplified surface conditions (A21)-(A24), with the above assumptions, are also 
employed. At the surface we require, in addition, a kinematic condition for the superfluid $\left(\hat{\rho}_{s} \neq 0\right.$ there) and this simply states that the superfluid and normal fluid share a common motion,

$$
v_{n}-v_{s}=\varepsilon\left(u_{n}-u_{s}\right) \frac{\partial \eta}{\partial x}
$$

The choice of incompressibility is really a device of expendiency: its relaxation does not alter the form of the nonlinear equation we obtain. The main effect of the exclusion of the healing terms, coupled with the incompressibility, is to replace $\hat{\rho}_{s}(y)$ by a constant. Finally, the relaxation terms are always sufficiently small (as $\varepsilon, \delta \rightarrow 0$, even for $\gamma \neq 0$ fixed) so as not to contribute at all in the far-field.

Since higher order terms are to be considered we must decide the relative size of $\varepsilon$ and $\delta$ : for example, in classical water-wave theory the Korteweg-de Vries equation is relevant when $\delta^{2}=O(\varepsilon)$. Examination of the various equations here leads us to first consider the obvious special case $\delta=O(\varepsilon)$, and so we choose $\delta=d \varepsilon$ where $d$ remains bounded (non-zero) as $\varepsilon \rightarrow 0$. (We shall comment on other possible choices later.) Further, since the nonlinearity is on the scale $O(\varepsilon)$ the far-field can be described by the variables $\mathcal{T}=\varepsilon t, \xi=x-c_{2} t$, where, by virtue of the linear theory, the wave propagates predominantly at the speed $c_{2}$. The speed $c_{1}$ does not appear explicitly here as the limit involves $\mu=O(1), \varepsilon \rightarrow 0$. All the dependent variables are assumed to have asymptotic expansions in integer powers of $\varepsilon$,

$$
Q \sim \sum_{n=0}^{\infty} \varepsilon^{n} Q_{n} \quad\left(Q \equiv u_{n}, u_{s}, v_{n}, v_{s}, r_{n}, r_{s}, p, \tau, \eta\right)
$$

and (see (45)),

$$
\varphi_{s}(\xi, \mathcal{T}, y ; \varepsilon)=\Theta_{0}(\xi, \mathcal{T})+{ }_{\varepsilon} \Theta_{1}(\xi, \mathcal{T})+O\left(\varepsilon^{2}\right) .
$$

The leading order terms yield the solution

$$
u_{n 0} \equiv 0, \quad p_{0}=\hat{\rho} \omega_{1}^{\prime} \eta_{0}, \quad \Theta_{0 \xi}=\frac{\omega_{1}^{\prime}}{c_{2}} \eta_{0}, \quad \tau_{01}=\frac{-\hat{a}_{T} \hat{\rho}_{s} \omega_{1}^{\prime}}{l \mu c_{2}} \eta_{0 \xi}
$$

for arbitrary $\eta_{0}(\xi, \mathcal{T})$ if

$$
c_{2}^{2}=\frac{\hat{\rho}_{s}}{\hat{\rho}} \omega_{1}^{\prime}\left(1-\frac{\hat{a}_{T}}{l}\right)
$$

which is (47) evaluated for $\hat{\rho}_{s}=$ consiant (and of course $\hat{a}_{T}$ is now also constant). The additional subscript ' 1 ' on $\tau_{0}$ denotes that this is the temperature perturbation evaluated at the surface. The results in (82) can be compared with those given in Section 4a, whence agreement is immediate if the incompressible limit is taken. 
The next order can be obtained in a straightforward manner, although we shall mention only a few of the intermediate steps. For example, we find that

$$
\begin{aligned}
& u_{n 1}=\frac{\lambda}{4 \alpha_{2}} \hat{\rho}_{n} \omega_{1}^{\prime}\left(y^{2}-2 y\right) \frac{\partial \eta_{0}}{\partial \xi}, \\
& p_{1}=\lambda\left(\alpha_{1}+\alpha_{2}\right) \frac{\partial v_{n 0}}{\partial y}+\hat{\rho}\left(\omega_{1}^{\prime} \eta_{0}+\frac{1}{2} \omega_{1}^{\prime \prime} \eta_{0}^{2}\right),
\end{aligned}
$$

with

$$
\hat{\rho} \hat{a}_{\rho_{s} \rho_{s}} r_{s 0}=-\frac{1}{2}\left(\Theta_{0 \xi}^{2}+2 \lambda \hat{\rho} \hat{a}_{\rho_{s}} \tau_{0}\right)
$$

and

$$
\frac{\partial^{2} v_{n 0}}{\partial y^{2}}-\frac{\left(\hat{\rho} \hat{a}_{T}\right)^{2}}{\lambda\left(\alpha_{1}+\alpha_{2}\right)} v_{n 0}=0 .
$$

This latter result is of some note: the structure of $v_{n 0}$ through the layer is governed by the length scale (in non-dimensional form),

$$
H=\kappa^{1 / 2}\left(\alpha_{1}+\alpha_{2}\right)^{1 / 2} /\left|\hat{\rho} \hat{a}_{T}\right|
$$

which is the well-known Clark thickness. This thickness is $O(1)$ (as $\varepsilon \rightarrow 0$ ), since all the parameters are assumed fixed in the limit process, and consequently 'Clark boundary layers' are not evident (see Roberts and Donnelly, [23]). Using the surface boundary conditions, which involve Taylor expansions about $y=1$, and eliminating the surface temperature perturbation $\tau_{11}$, we obtain the equation for $\eta_{0}$,

$$
2 l \hat{\rho} \frac{\partial \eta_{0}}{\partial \mathcal{T}}+\left[\hat{\rho}_{s}\left(l-\hat{a}_{T}\right)\left(2 \omega_{1}^{\prime}+\omega_{1}^{\prime \prime}\right)+\left(\hat{a}_{\rho_{s} T} / \hat{a}_{\rho_{s} \rho_{s}}\right) \omega_{1}^{\prime 2}\right] \frac{\eta_{0}}{c_{2}} \frac{\partial \eta_{0}}{\partial \xi}=d k \frac{\partial^{2} \eta_{0}}{\partial \xi^{2}},
$$

where $k(>0)$ is a complicated constant involving thermodynamic terms ( $\hat{a}_{T}$ etc.) and $l, \hat{\rho}, \hat{\rho}_{s}, \mu, \kappa, \alpha_{1}, \alpha_{2}$. To offer a simplified version of this equation let us suppose that $l$ is large (a point we made in Sections $4 a$ and $4 c$ ), whence

$$
\begin{aligned}
& 2 \hat{\rho} \frac{\partial \eta_{0}}{\partial \mathscr{T}}+\frac{\hat{\rho}_{s}}{c_{2}}\left(2 \omega_{1}^{\prime}+\omega_{1}^{\prime \prime}\right) \eta_{0} \frac{\partial \eta_{0}}{\partial \xi} \\
&=d\left[\frac{2}{3 \alpha_{2}} \hat{\rho}_{n}^{2} \omega_{1}^{\prime}+\hat{\rho}_{s}\left(-\hat{a}_{T}\right)\left(\frac{\alpha_{1}+\alpha_{2}}{\kappa}\right)^{1 / 2} \operatorname{coth}\left(H^{-1}\right)\right] \frac{\partial^{2} \eta_{0}}{\partial \xi^{2}} .
\end{aligned}
$$

Equation (83) - and its simplified version (84)-is a Burgers equation with a positive diffusivity (this is clear from (84)) and so solutions for $\eta_{0}$ either decay or approach the steady shock (or 'Taylor') profile. Since we are more concerned with initial profiles which tend to zero as $\xi \rightarrow \pm \infty$ (or are possibly oscillatory) we see 
that no such profiles can propagate unchanged. In particular no solitary wave solution exists nor are solitons produced from fairly general initial data: this has been suggested by Huberman [12] and Nakajima et al. [20] on the basis that the relevant equation is the Korteweg-de Vries equation. The difficulty really stems from the character of the normal fluid for which the viscous terms dominate to produce zero normal flow to leading order. As our equation (84) amply demonstrates, the neglect of the viscous terms (i.e. $\alpha_{1}, \alpha_{2} \rightarrow 0$ ) is in fact a singular limit and any argument which therefore ignores the motion of the normal fluid altogether will be open to criticism. Of course we have used a special set of equations and a well-defined limit $\delta, \varepsilon \rightarrow 0$ (with all other parameters fixed) but, we claim, more sophisticated models (like A1-A24) would not alter the form of (83) and the limiting process is the only one consistent for small amplitude long waves. However it is clear that if all the parameters were treated as functions of $\varepsilon$ then a great variety of equations could presumably be concocted to replace (83), by suitable choice of the $\varepsilon$-dependence. Although such a procedure is not legitimate on physical grounds (as we argued in Section 1), it is quite in order as a mathematical exercise: we shall pursue this line in Section $5 b$.

The analysis leading to equation (83) was based on the special choice $\delta=O(\varepsilon)$ and it might be expected that other choices would lead to other nonlinear evolution equations. In a sense this is correct, but the only real alternative is equation (83) with the nonlinear term absent i.e. the heat conduction equation (which of course has decaying solutions). To see this we first note that the transformation

$$
x \rightarrow \frac{\delta}{\varepsilon} x, \quad t \rightarrow \frac{\delta}{\varepsilon} t, \quad v_{q} \rightarrow \frac{\varepsilon}{\delta} v_{q} \quad(q \equiv n, s), \quad \tau \rightarrow \frac{\varepsilon}{\delta} \tau, \quad \varphi_{s} \rightarrow \frac{\delta}{\varepsilon} \varphi_{s},
$$

is equivalent to replacing $\delta$ by $\varepsilon$. Thus there exists a time and distance scale, for arbitrary $\delta$, on which the Burgers equation is valid. On the other hand we could argue that, if $\varepsilon=o(\delta)$, then with $\mathcal{T}=\delta t$ and $\xi=O(1)$ we obtain the heat conduction equation which in turn leads to (83) on longer time and distance scales. Correspondingly, if $\delta=o(\varepsilon)$, then with $\mathcal{T}=\varepsilon t$ and $\xi=O(1)$ we obtain the nonlinear wave equation (i.e. (83) with $d=0$ ), however as the solutions of this equation steepen there is an appropriate (shorter) length scale for which (83) is again recovered. It would therefore appear that the Burgers equation is the generic equation for the evolution of a small amplitude wave in third sound.

\section{b. A Korteweg-de Vries theory}

There has been much interest over the last decade or so in 'soliton' theory and it behoves us to consider the possibility of a Korteweg-de Vries (KdV) equation occurring here. As we have already seen this can only be accomplished at the expense of treating some (or all) of the parameters as functions of $\varepsilon$. A fairly cursory inspection of the governing equations (as used in Section 5a) indicates 
that a term $\eta_{0 \xi \xi \xi}$ can arise if we account for the transfer of heat along the layer i.e. from the term $\tau_{x x}$ in the energy equation. Since third sound involves a heat imbalance between peaks and troughs we can anticipate that any heat transfer along the layer will tend to disperse the wave. To incorporate this effect we use exactly the governing equations discussed in Section $5 \mathrm{a}$, but for convenience set $\tau=\tilde{\tau} / \mu$, and then choose $\kappa / \mu=O\left(\varepsilon^{-1}\right), \mu=O(1)$ or larger. We shall also use $\delta-d \varepsilon$, as before.

The variables are once again

$$
\widetilde{J}=\varepsilon t, \quad \xi=x-c_{2} t,
$$

and the expansions also follow the same form with the exception that now

$$
\tilde{\tau} \sim \tilde{\tau}_{0}(\xi, \mathscr{T})+\sum_{n=1}^{\infty} \varepsilon^{n} \tilde{\tau}_{n}(\xi, \mathscr{T}, y)
$$

To be precise about our assumption for $\kappa$ we write $\kappa / \mu=\mathfrak{N} / \varepsilon$ and the leading order is just

$$
p_{0}=\hat{\rho} \omega_{1}^{\prime} \eta_{0}, \quad \Theta_{0 \xi}=\frac{\omega_{1}^{\prime}}{c_{2}} \eta_{0}, \quad \tilde{\tau}_{0}=-\hat{\rho}_{s}\left(\frac{a_{T} \omega_{1}^{\prime}}{l c_{2}}\right) \eta_{0 \xi},
$$

(see (82)) and $c_{2}$ is the same. At the next order rather more significant modifications occur resulting in $v_{\text {noyy }}=0$, whence the Clark thickness is not relevant, but otherwise the calculation is very similar to the earlier case. The equation for $\eta_{0}(\xi, \mathscr{T})$ is now determined as

$$
\begin{aligned}
2 l \hat{\rho} \frac{\partial \eta_{0}}{\partial \mathscr{T}}+\left[( l - \hat { a } _ { T } ) \left(2 \omega_{1}^{\prime}+\right.\right. & \left.\left.\omega_{1}^{\prime \prime}\right) \frac{\hat{\rho}_{s}}{c_{2}}+\frac{\hat{a}_{\rho_{s} T} \omega_{1}^{\prime 2}}{\hat{a}_{\rho_{s} \rho s} c_{2}}\right] \eta_{0} \frac{\partial \eta_{0}}{\partial \xi} \\
& +d^{2} \Re \hat{\rho}_{s}\left(\frac{-\hat{a}_{T} \omega_{1}^{\prime}}{l c_{2}}\right) \frac{\partial^{3} \eta_{0}}{\partial \xi^{3}}=d k^{\prime} \frac{\partial^{2} \eta_{0}}{\partial \xi^{2}}
\end{aligned}
$$

where $k^{\prime}$ is a slight variation on $k$ (see (83)) due essentially to the form of $v_{n 0}$ here. Equation (85) is a Korteweg-de Vries-Burgers equation (which has some relevance to the undular bore in water-wave theory; see Johnson [14]). However, even though we have demonstrated that both dissipative and dispersive terms can arise, our main interest is in the appearance of a $\mathrm{KdV}$ equation. If we retain the same definitions of $\mathcal{T}$ and $\xi$, but consider $\delta=o(\varepsilon)$ with $d^{2} \Re=O(1)$, then the dissipative term is absent in (85) (equivalently, set $k^{\prime}=0$ ). (It is fairly easy to see that we do indeed obtain (85), with $k^{\prime}=0$, by assuming $\delta=o(\varepsilon)$ and $\kappa / \mu=$ $O\left(\varepsilon \delta^{-2}\right)$ in the original equations.)

It must be emphasized that our procedure borders on the ad hoc and is very little more than an exercise in choosing parameter and scaling sizes. As we have argued earlier, this approach is in contradiction to the nature of the physical 
system. For a given helium II layer the parameters $\kappa, \mu$ etc. are fixed and certainly cannot depend on the amplitude and wavelength of the third sound under examination. However it is possible to put forward a case, based on the numerical size of various parameters, that a $\mathrm{KdV}$ equation might be relevant if $\varepsilon$ and $\delta$ are small and the thermal conductivity, $\kappa$, is large. If this underlying premise is accepted then our theory gives, explicitly, the coefficients of the KdV equation in terms of the thermodynamic and other physical constants. In particular the ratio (dispersive term)/(time derivative term) is just

$$
\frac{1}{2} \frac{\kappa}{\mu} \frac{\hat{\rho}_{s}}{\hat{\rho}}\left(\frac{-\hat{a}_{T} \omega_{1}^{\prime}}{l^{2} c_{2}}\right),
$$

if we ignore the dependence on $\varepsilon, \delta$. Further, if we choose the non-dimensionalisation so that $\hat{\rho}=c_{2}=1$ (which we may always do), then this ratio becomes

$$
\frac{1}{2} \frac{\kappa}{\mu l}\left(-\frac{\hat{a}_{T}}{l}\right)\left(1-\frac{\hat{a}_{T}}{l}\right)^{-1}
$$

and dispersion is presumably therefore more important the larger the value of (86). (Note that since $\hat{a}_{T}<0,(86)$ is positive and so conforms with the usual sign convention ('positive' dispersion) in the $\mathrm{KdV}$ equation.)

\section{Conclusion}

In this article we have developed a number of theories for third sound by constructing asymptotic solutions to various sets of governing equations. It has been shown, using this fairly systematic approach, that we can isolate different aspects of the problem. So, for example, it has been possible to extract the phenomenon of a wave hierarchy on the one hand and the effects of healing on the other. However, underlying all the presentations is the unavoidable-and rather unpalatable - fact that the mathematical problem is quite involved even for some of the simpler models. Nevertheless it is evident that the formal procedure we have adopted avoids many of the pitfalls inherent in tackling the problem in a purely ad hoc manner. In fact, with the equations as presented in the Appendix, it is now feasible to examine any particular special case of interest. In consequence it is hoped that the assumptions and error terms will be clearly understood. It is also possible to formulate the exact problem, with the vapour, and use just the limit $\varepsilon, \delta \rightarrow 0$; we have avoided this mainly because of the difficulties encountered with the healing terms involving the necessity for numerical solutions (see Section 4c). The overall impression is that the two-fluid equations, with healing, relaxation and a vapour, embody a very large amount of detailed physics which is worthy of further study. Of course the aim must be to test any of the special 
predictions against the observed behaviour of helium II, but the results to-date would seem to indicate that a two-fluid continuum model is amazingly successful, even for very thin layers. Certainly all the strengths and weaknesses of these various models must be examined with some care before they are rejected as being unacceptable. For example, we can anticipate that any continuum theory is likely to fail when the superfluid is as little as one atomic layer in depth. The real surprise is that the equations appear to remain quite reasonable at about two atomic layers thickness.

We have chosen to look at some linear and nonlinear theories for third sound, of varying complexity, mainly to demonstrate the wealth of detail (and correspondence between differing models) that is available. The linear theories by and large agree with much of the earlier work and indicate how the derivations can be generalised, or other physical phenomena included. The nonlinear theory developed in Section 5a, leading to the Burgers equation, shows that dissipative rather than dispersive effects dominate in the propagation of small amplitude long waves. However, more or less as a mathematical exercise and to keep pace with the current vogue, we have obtained a $\mathrm{KdV}$ equation by imposing a special relation between the thermal conductivity, $\kappa$, and $\varepsilon$. It is just possible, under very special circumstances, that the assumptions required for the $\mathrm{KdV}$ equation to pertain are realisable in practice.

To conclude, and to suggest that the work presented here is no more than a beginning, let us mention two or three other problems that could be examined as an extension of the study of third sound. The discussion of the wave hierarchy, and in particular the variation of $c_{1}$ and $c_{2}$ with $h$ (Figure 2), suggests that the whole analysis should be re-considered with $c_{1}-c_{2}$ small e.g. $c_{1}-c_{2}=O(\varepsilon)$. It is well-known that in this type of problem the nonlinear term (and higher order dissipative and dispersive terms) become important and might therefore significantly alter the character of the propagation on very thin films. As we saw in Section $4 \mathrm{c}$ the problem of the healing terms gives rise to complicated equations for $\hat{\rho}_{s}(y)$ and $r_{s}$ : there is every likelihood that considerable (analytical) headway can be made if the liquid is assumed to be incompressible, or nearly so. Finally, also in connection with the rôle of the superfluid density, the equations can be used to test the possibility of employing specific models for the Helmholtz free energy, $A\left(\rho, \rho_{s}, T\right)$. So for example, particularly if the healing terms are retained, we could try $A=A_{1}(\rho, T)+A_{2}(\rho, T)\left[\rho_{s}-\bar{\rho}_{s}(\rho, T)\right]^{2}$ where $\bar{\rho}_{s}$ is the equilibrium superfluid density. Actually even this could be simplified further if the liquid were again assumed to be incompressible ( $\rho=$ constant): then $\rho_{s}=\rho_{s}(y)$ by virtue of the healing terms above. There are undoubtably many other models or assumptions that can be tested against the general equations presented here, and incorporated into a theory of third sound. 


\section{Appendix}

The complete set of equations and boundary conditions, written in non-dimensional form, are given here.

\section{Liquid model:}

$$
\begin{aligned}
& \frac{\partial r}{\partial t}+\frac{\partial}{\partial x}\left[u_{n}\left(\hat{\rho}_{n}+\varepsilon r_{n}\right)+u_{s}\left(\hat{\rho}_{s}+\varepsilon r_{s}\right)\right]+\frac{\partial}{\partial y}\left[v_{n}\left(\hat{\rho}_{n}+\varepsilon r_{n}\right)+v_{s}\left(\hat{\rho}_{s}+\varepsilon r_{s}\right)\right]=0, \\
& \frac{\partial}{\partial t}\left[u_{n}\left(\hat{\rho}_{n}+\varepsilon r_{n}\right)+u_{s}\left(\hat{\rho}_{s}+\varepsilon r_{s}\right)\right]+\varepsilon \frac{\partial}{\partial x}\left[u_{n}^{2}\left(\hat{\rho}_{n}+\varepsilon r_{n}\right)+u_{s}^{2}\left(\hat{\rho}_{s}+\varepsilon r_{s}\right)\right] \\
& +\varepsilon \frac{\partial}{\partial y}\left[u_{n} v_{n}\left(\hat{\rho}_{n}+\varepsilon r_{n}\right)+u_{s} v_{s}\left(\hat{\rho}_{s}+\varepsilon r_{s}\right)\right] \\
& =-\frac{\partial p}{\partial x}-\varepsilon \delta^{2} \beta \frac{\partial}{\partial x}\left[\frac{\left(\partial r_{s} / \partial x\right)^{2}}{\hat{\rho}_{s}+\varepsilon r_{s}}\right]-\beta \frac{\partial}{\partial y}\left[\frac{\partial r_{s} / \partial x\left(d \hat{\rho}_{s} / d y+\varepsilon \partial r_{s} / \partial y\right)}{\hat{\rho}_{s}+\varepsilon r_{s}}\right] \\
& \quad+\delta\left(\alpha_{1}+\alpha_{2}\right) \frac{\partial^{2} u_{n}}{\partial x^{2}}+\frac{1}{2} \frac{\alpha_{2}}{\delta} \frac{\partial^{2} u_{n}}{\partial y^{2}}+\delta\left(\alpha_{1}+\frac{1}{2} \alpha_{2}\right) \frac{\partial^{2} v_{n}}{\partial x \partial y},
\end{aligned}
$$

$$
\begin{aligned}
& \varepsilon \delta^{2}\left\{\frac{\partial}{\partial t}\left[v_{n}\left(\hat{\rho}_{n}+\varepsilon r_{n}\right)+v_{s}\left(\hat{\rho}_{s}+\varepsilon r_{s}\right)\right]+\varepsilon \frac{\partial}{\partial x}\left[u_{n} v_{n}\left(\hat{\rho}_{n}+\varepsilon r_{n}\right)+u_{s} v_{s}\left(\hat{\rho}_{s}+\varepsilon r_{s}\right)\right]\right. \\
& \left.+\varepsilon \frac{\partial}{\partial y}\left[v_{n}^{2}\left(\hat{\rho}_{n}+\varepsilon r_{n}\right)+v_{s}^{2}\left(\hat{\rho}_{s}+\varepsilon r_{s}\right)\right]\right\} \\
& =-\frac{d \hat{p}}{d y}-\varepsilon \frac{\partial p}{\partial y}-(\hat{p}+\varepsilon r) \frac{d \omega}{d y}-\varepsilon \delta^{2} \beta \frac{\partial}{\partial x}\left[\frac{\partial r_{s} / \partial x\left(d \hat{\rho}_{s} / d y+\varepsilon \partial r_{s} / \partial y\right)}{\hat{\rho}_{s}+\varepsilon r_{s}}\right] \\
& -\beta \frac{\partial}{\partial y}\left[\frac{\left(d \hat{\rho}_{s} / d y+\varepsilon \partial r_{s} / \partial y\right)^{2}}{\hat{\rho}_{s}+\varepsilon r_{s}}\right]+\varepsilon \delta\left(\alpha_{1}+\alpha_{2}\right) \frac{\partial^{2} v_{n}}{\partial y^{2}} \\
& +\frac{1}{2} \varepsilon \delta^{3} \alpha_{2} \frac{\partial^{2} v_{n}}{\partial x^{2}}+\varepsilon \delta\left(\alpha_{1}+\frac{1}{2} \alpha_{2}\right) \frac{\partial^{2} u_{n}}{\partial x \partial y},
\end{aligned}
$$

$$
\begin{aligned}
& \left.\left(a+\rho \frac{\partial a}{\partial \rho}\right)\right|_{\substack{\hat{\rho}+\varepsilon r \\
\hat{\rho}_{s}+\varepsilon r_{s} \\
1+\delta \varepsilon \tau}}+\omega+\varepsilon \frac{\partial \varphi_{s}}{\partial t}+\frac{1}{2} \varepsilon^{2}\left[u_{s}^{2}+\delta^{2} v_{s}^{2}-\left(u_{n}-u_{s}\right)^{2}-\delta^{2}\left(v_{n}-v_{s}\right)^{2}\right] \\
& -\frac{\varepsilon \delta \gamma}{\sqrt{ }\left(\hat{\rho}_{s}+\varepsilon r_{s}\right)}\left\{\frac{\partial r_{s}}{\partial t}+\frac{\partial}{\partial x}\left[u_{s}\left(\hat{\rho}_{s}+\varepsilon r_{s}\right)\right]+\frac{\partial}{\partial y}\left[v_{s}\left(\hat{\rho}_{s}+\varepsilon r_{s}\right)\right]\right\}=0, \\
& \hat{p}+\varepsilon p=(\hat{\rho}+\varepsilon r)^{2} \frac{\partial a}{\partial \rho}+(\hat{\rho}+\varepsilon r)\left(\hat{\rho}_{s}+\varepsilon r_{s}\right) \frac{\partial a}{\partial \rho_{s}} \\
& -\varepsilon \delta^{2} \beta \frac{\partial^{2} r_{s}}{\partial x^{2}}-\beta\left(\frac{d^{2} \hat{\rho}_{s}}{d y^{2}}+\varepsilon \frac{\partial^{2} r_{s}}{\partial y^{2}}\right),
\end{aligned}
$$




$$
\begin{aligned}
(\hat{p}+ & \varepsilon r) \frac{\partial a}{\partial \rho_{s}}+\frac{1}{2} \varepsilon^{2}\left[\left(u_{n}-u_{s}\right)^{2}+\delta^{2}\left(v_{n}-v_{s}\right)^{2}\right] \\
& -\frac{\beta}{\left(\hat{\rho}_{s}+\varepsilon r_{s}\right)}\left[\frac{d^{2} \hat{\rho}_{s}}{d y^{2}}+\varepsilon \frac{\partial^{2} r_{s}}{\partial y^{2}}+\varepsilon \delta^{2} \frac{\partial^{2} r_{s}}{\partial x^{2}}\right] \\
& +\frac{1}{2} \frac{\beta}{\left(\hat{\rho}_{s}+\varepsilon r_{s}\right)^{2}}\left[\left(\frac{d \hat{\rho}_{s}}{d y}+\varepsilon \frac{\partial r_{s}}{\partial y}\right)^{2}+\varepsilon^{2} \delta^{2}\left(\frac{\partial r_{s}}{\partial x}\right)^{2}\right] \\
& +\frac{\varepsilon \delta \gamma}{\sqrt{ }\left(\hat{\rho}_{s}+\varepsilon r_{s}\right)}\left\{\frac{\partial r_{s}}{\partial t}+\frac{\partial}{\partial x}\left[u_{s}\left(\hat{\rho}_{s}+\varepsilon r_{s}\right)\right]+\frac{\partial}{\partial y}\left[v_{s}\left(\hat{\rho}_{s}+\varepsilon r_{s}\right)\right]\right\}=0, \\
(1+ & \delta \varepsilon \tau)\left\{\frac{\partial}{\partial t}\left[(\hat{\rho}+\varepsilon r) \frac{\partial a}{\partial T}\right]+\varepsilon \frac{\partial}{\partial x}\left[u_{n}(\hat{\rho}+\varepsilon r) \frac{\partial a}{\partial T}\right]+\varepsilon \frac{\partial}{\partial y}\left[v_{n}(\hat{\rho}+\varepsilon r) \frac{\partial a}{\partial T}\right]\right\} \\
& +\frac{\varepsilon^{2} \delta \gamma}{\sqrt{ }\left(\hat{\rho}_{s}+\varepsilon r_{s}\right)}\left\{\frac{\partial r_{s}}{\partial t}+\frac{\partial}{\partial x}\left[u_{s}\left(\hat{\rho}_{s}+\varepsilon r_{s}\right)\right]+\frac{\partial}{\partial y}\left[v_{s}\left(\hat{\rho}_{s}+\varepsilon r_{s}\right)\right]\right\}^{2} \\
& +\varepsilon^{2} \delta \alpha_{1}\left(\frac{\partial u_{n}}{\partial x}+\frac{\partial v_{n}}{\partial y}\right)^{2}+\varepsilon^{2} \delta \alpha_{2}\left[\left(\frac{\partial u_{n}}{\partial x}\right)^{2}+\left(\frac{\partial v_{n}}{\partial y}\right)^{2}\right] \\
& +\frac{1}{2} \frac{\varepsilon^{2}}{\delta} \alpha_{2}\left(\frac{\partial u_{n}}{\partial y}+\delta^{2} \frac{\partial v_{n}}{\partial x}\right)^{2}+\varepsilon \delta^{2} \kappa\left(\frac{\partial^{2} \tau}{\partial x^{2}}+\frac{1}{\delta^{2}} \frac{\partial^{2} \tau}{\partial y^{2}}\right)=0, \\
u_{n}=v_{n}=v_{s}=0 \text { on } y=0, & \text { (A })^{2} / \partial y=0 \text { on } y=0 .
\end{aligned}
$$

\section{Vapour model:}

$$
\begin{aligned}
& \frac{1}{\Delta} \frac{\partial r_{g}}{\partial t}+\frac{\partial}{\partial x}\left[u_{g}\left(\hat{\rho}_{g}+\varepsilon r_{g}\right)\right]+\frac{\partial}{\partial y}\left[v_{g}\left(\hat{\rho}_{g}+\varepsilon r_{g}\right)\right]=0 \\
& \frac{1}{\Delta} \frac{\partial}{\partial t}\left[u_{g}\left(\hat{\rho}_{g}+\varepsilon r_{g}\right)\right]+\varepsilon \frac{\partial}{\partial x}\left[u_{g}^{2}\left(\hat{\rho}_{g}+\varepsilon r_{g}\right)\right]+\varepsilon \frac{\partial}{\partial y}\left[u_{g} v_{g}\left(\hat{\rho}_{g}+\varepsilon r_{g}\right)\right] \\
& =-\frac{\partial p_{g}}{\partial x}+\delta\left(\alpha_{1 g}+\alpha_{2 g}\right) \frac{\partial^{2} u_{g}}{\partial x^{2}}+\frac{1}{2} \frac{\alpha_{2 g}}{\delta} \frac{\partial^{2} u_{g}}{\partial y^{2}}+\delta\left(\alpha_{1 g}+\frac{1}{2} \alpha_{2 g}\right) \frac{\partial^{2} v_{g}}{\partial x \partial y} \\
& \varepsilon \delta^{2}\left\{\frac{1}{\Delta} \frac{\partial}{\partial t}\left[v_{g}\left(\hat{\rho}_{g}+\varepsilon r_{g}\right)\right]+\varepsilon \frac{\partial}{\partial x}\left[u_{g} v_{g}\left(\hat{\rho}_{g}+\varepsilon r_{g}\right)\right]+\varepsilon \frac{\partial}{\partial y}\left[v_{g}^{2}\left(\hat{\rho}_{g}+\varepsilon r_{g}\right)\right]\right\} \\
& =-\frac{d \hat{p}_{g}}{d y}-\varepsilon \frac{\partial p_{g}}{\partial y}-\left(\hat{\rho}_{g}+\varepsilon r_{g}\right) \frac{1}{\Delta^{2}} \frac{d \omega}{d y} \\
& \quad+\varepsilon \delta\left(\alpha_{1 g}+\alpha_{2 g}\right) \frac{\partial^{2} v_{g}}{\partial y^{2}}+\frac{1}{2} \varepsilon \delta^{3} \alpha_{2 g} \frac{\partial^{2} v_{g}}{\partial x^{2}}+\varepsilon \delta\left(\alpha_{1 g}+\frac{1}{2} \alpha_{2 g}\right) \frac{\partial^{2} u_{g}}{\partial x \partial y}
\end{aligned}
$$




$$
\begin{gathered}
\hat{p}_{g}+\varepsilon p_{g}=\left(\hat{\rho}_{g}+\varepsilon r_{g}\right)^{2} \frac{\partial a_{g}}{\partial \rho} \\
\left(1+\varepsilon \delta \tau_{g}\right)\left\{\frac{1}{\Delta} \frac{\partial}{\partial t}\left[\left(\hat{\rho}_{g}+\varepsilon r_{g}\right) \frac{\partial a_{g}}{\partial T}\right]\right. \\
\left.+\varepsilon \frac{\partial}{\partial x}\left[u_{g}\left(\hat{\rho}_{g}+\varepsilon r_{g}\right) \frac{\partial a_{g}}{\partial T}\right]+\varepsilon \frac{\partial}{\partial y}\left[v_{g}\left(\hat{\rho}+\varepsilon r_{g}\right) \frac{\partial a_{g}}{\partial T}\right]\right\} \\
+\varepsilon^{2} \delta \alpha_{1 g}\left(\frac{\partial u_{g}}{\partial x}+\frac{\partial v_{g}}{\partial y}\right)^{2}+\varepsilon^{2} \delta \alpha_{2 g}\left[\left(\frac{\partial u_{g}}{\partial x}\right)^{2}+\left(\frac{\partial v_{g}}{\partial y}\right)^{2}\right] \\
+\frac{1}{2} \frac{\varepsilon^{2}}{\delta} \alpha_{2 g}\left(\frac{\partial u_{g}}{\partial y}+\delta^{2} \frac{\partial v_{g}}{\partial x}\right)^{2}+\varepsilon \kappa_{g}\left(\delta^{2} \frac{\partial^{2} \tau_{g}}{\partial x^{2}}+\frac{\partial^{2} \tau_{g}}{\partial y^{2}}\right)=0
\end{gathered}
$$

Jump conditions (on $y=1+\varepsilon \eta$ ):

$$
\begin{gathered}
j / \cos \theta=\varepsilon \delta(\hat{\rho}+\varepsilon r)\left(v_{n}-\frac{\partial \eta}{\partial t}-\varepsilon u_{n} \frac{\partial \eta}{\partial x}\right) \\
=\varepsilon \delta \Delta \sigma\left(\hat{\rho}_{g}+\varepsilon r_{g}\right)\left(v_{g}-\frac{1}{\Delta} \frac{\partial \eta}{\partial t}-\varepsilon u_{g} \frac{\partial \eta}{\partial x}\right), \quad \text { (A15) } \\
\hat{p}+\varepsilon p-\sigma \Delta^{2}\left(\hat{p}_{g}+\varepsilon p_{g}\right)-\varepsilon \delta \alpha_{1}\left(\frac{\partial u_{n}}{\partial x}+\frac{\partial v_{n}}{\partial y}\right)+\varepsilon \delta \Delta \sigma^{2} \alpha_{1 g}\left(\frac{\partial u_{g}}{\partial x}+\frac{\partial v_{g}}{\partial y}\right) \\
+\varepsilon \delta \cos ^{2} \theta\left[\sigma \Delta^{2} \alpha_{2 g}\left(\frac{\partial v_{g}}{\partial y}+\left(\varepsilon \delta \frac{\partial \eta}{\partial x}\right)^{2} \frac{\partial u_{g}}{\partial x}-\varepsilon \frac{\partial \eta}{\partial x}\left(\frac{\partial u_{g}}{\partial y}+\delta^{2} \frac{\partial v_{g}}{\partial x}\right)\right\}\right. \\
-j^{2}\left(\frac{1}{\hat{\rho}+\varepsilon r}-\frac{1}{\sigma} \frac{1}{\hat{\rho}_{g}+\varepsilon r_{g}}\right)+\left(\frac{\beta \cos ^{2} \theta}{\hat{\rho}_{s}+\varepsilon r_{s}}\right)\left(\frac{d \hat{\rho}_{s}}{\partial y}+\varepsilon \frac{\partial r_{s}}{\partial y}-\varepsilon^{2} \delta^{2} \frac{\partial \eta}{\partial x} \frac{\partial r_{s}}{\partial x}\right)^{2}=0 \\
\varepsilon\left[\alpha_{2}\left\{2 \varepsilon \delta \frac{\partial \eta}{\partial x}\left(\frac{\partial u_{n}}{\partial x}-\frac{\partial v_{n}}{\partial y}\right)-\left(1-\left(\varepsilon \delta \frac{\partial \eta}{\partial x}\right)^{2}\right)\left(\frac{\partial u_{n}}{\partial y}+\delta^{2} \frac{\partial v_{n}}{\partial x}\right)\right\}\right. \\
\left.-\Delta^{2} \sigma \alpha_{2 g}\left\{2 \varepsilon \delta \frac{\partial \eta}{\partial x}\left(\frac{\partial u_{g}}{\partial x}-\frac{\partial v_{g}}{\partial y}\right)-\left(1-\left(\varepsilon \delta \frac{\partial \eta}{\partial x}\right)^{2}\right)\left(\frac{\partial u_{g}}{\partial y}+\delta^{2} \frac{\partial v_{g}}{\partial x}\right)\right\}\right] \\
+\frac{\varepsilon \delta \beta}{\hat{\rho}_{s}+\varepsilon r_{s}}\left[\frac{\partial r_{s}}{\partial x}+\frac{\partial \eta}{\partial x}\left(\frac{d \hat{\rho}_{s}}{d y}+\varepsilon \frac{\partial r_{s}}{\partial y}\right)\right]\left(\frac{\hat{\rho}_{s}}{d y}+\varepsilon \frac{\partial r_{s}}{\partial y}-\varepsilon^{2} \delta^{2} \frac{\partial \eta}{\partial x} \frac{\partial r_{s}}{\partial x}\right)=0,
\end{gathered}
$$




$$
\begin{aligned}
& {\left[\psi-(1+\varepsilon \delta \tau)\left(\frac{\partial a}{\partial T}-\Delta^{2} \frac{\partial a_{g}}{\partial T}\right)\right] j / \cos \theta} \\
& =\varepsilon \delta\left[\frac{\partial}{\partial y}\left(\kappa \tau-\Delta^{3} \sigma \kappa_{g} \tau_{g}\right)-\varepsilon \delta^{2} \frac{\partial \eta}{\partial x} \frac{\partial}{\partial x}\left(\kappa \tau-\Delta^{3} \sigma \kappa_{g} \tau_{g}\right)\right],
\end{aligned}
$$

where

$$
\begin{aligned}
& \psi= a+\frac{\hat{p}+\varepsilon p}{\hat{\rho}+\varepsilon r}-\Delta^{2}\left(a_{g}+\frac{\hat{\rho}_{g}+\varepsilon p_{g}}{\hat{\rho}_{g}+\varepsilon r_{g}}\right)+\frac{1}{2} \varepsilon^{2}\left[u_{n}^{2}+\delta^{2} v_{n}^{2}-\Delta^{2}\left(u_{g}^{2}+\delta^{2} v_{g}^{2}\right)\right] \\
&+\frac{1}{2} \varepsilon^{2} \frac{\hat{\rho}_{s}+\varepsilon r_{s}}{\hat{\rho}+\varepsilon r}\left[\left(u_{n}-u_{s}\right)^{2}+\delta^{2}\left(v_{n}-v_{s}\right)^{2}\right] \\
&-\varepsilon \delta j \cos \theta \frac{\partial \eta}{\partial t}\left[\frac{1}{\hat{\rho}+\varepsilon r}-\frac{1}{\sigma} \frac{1}{\hat{\rho}_{g}+\varepsilon r_{g}}\right] \\
&+\frac{\varepsilon \delta \gamma /\left(\hat{\rho}_{s}+\varepsilon r_{s}\right)}{\hat{\rho}+\varepsilon r}\left\{\frac{1}{\Delta} \frac{\partial r_{s}}{\partial t}+\frac{\partial}{\partial x}\left[u_{s}\left(\hat{\rho}_{s}+\varepsilon r_{s}\right)\right]+\frac{\partial}{\partial y}\left[v_{s}\left(\hat{\rho}_{s}+\varepsilon r_{s}\right)\right]\right\} \\
&-\varepsilon \delta\left[\frac{\alpha_{1}}{\hat{\rho}+\varepsilon r}\left(\frac{\partial u_{n}}{\partial x}+\frac{\partial v_{n}}{\partial y}\right)-\frac{\Delta^{2} \alpha_{1 g}}{\hat{\rho}_{g}+\varepsilon r_{g}}\left(\frac{\partial u_{g}}{\partial x}+\frac{\partial v_{g}}{\partial y}\right)\right] \\
&+\frac{\beta \cos ^{2} \theta}{2(\hat{\rho}+\varepsilon r)\left(\hat{\rho}_{s}+\varepsilon r_{s}\right)}\left[\frac{\partial \hat{\rho}_{s}}{d y}+\varepsilon \frac{\partial r_{s}}{\partial y}-\varepsilon^{2} \delta^{2} \frac{\partial \eta}{\partial x} \frac{\partial r_{s}}{\partial x}\right]^{2} \\
&-\varepsilon \delta \cos ^{2} \theta\left\{\frac{\alpha_{2}}{\hat{\rho}+\varepsilon r}\left[\frac{\partial v_{n}}{\partial y}+\left(\varepsilon \delta \frac{\partial \eta}{\partial x}\right)^{2} \frac{\partial u_{n}}{\partial x}-\varepsilon \frac{\partial \eta}{\partial x}\left(\frac{\partial u_{n}}{\partial y}+\delta^{2} \frac{\partial v_{n}}{\partial x}\right)\right]\right. \\
&(\mathrm{Al} / 9) \\
&\left.-\frac{\Delta^{2} \alpha_{2 g}}{\hat{\rho}_{g}+\varepsilon r_{g}}\left[\frac{\partial v_{g}}{\partial y}+\left(\varepsilon \delta \frac{\partial \eta}{\partial x}\right)^{2} \frac{\partial u_{g}}{\partial x}-\varepsilon \frac{\partial \eta}{\partial x}\left(\frac{\partial u_{g}}{\partial y}+\delta^{2} \frac{\partial v_{g}}{\partial x}\right)\right]\right\}
\end{aligned}
$$

Simplified boundary conditions (on $y=1+\varepsilon \eta$ ):

$$
\begin{aligned}
& \hat{p}+\varepsilon p-\varepsilon \delta \alpha_{1}\left(\frac{\partial u_{n}}{\partial x}+\frac{\partial v_{n}}{\partial y}\right) \\
& +\cos ^{2} \theta\left\{\frac{\beta}{\hat{\rho}_{s}+\varepsilon r_{s}}\left[\frac{d \hat{\rho}_{s}}{d y}+\varepsilon \frac{\partial r_{s}}{\partial x}-\varepsilon^{2} \delta^{2} \frac{\partial \eta}{\partial x} \frac{\partial r_{s}}{\partial x}\right]^{2}\right. \\
& \left.\quad-\varepsilon \delta \alpha_{2}\left[\frac{\partial v_{n}}{\partial y}+\left(\varepsilon \delta \frac{\partial \eta}{\partial x}\right)^{2} \frac{\partial u_{n}}{\partial x}-\varepsilon \frac{\partial \eta}{\partial y}\left(\frac{\partial u_{n}}{\partial y}+\delta^{2} \frac{\partial v_{n}}{\partial x}\right)\right]\right\}=p_{v},
\end{aligned}
$$




$$
\begin{gathered}
{\left[1-\left(\varepsilon \delta \frac{\partial \eta}{\partial x}\right)^{2}\right]\left(\frac{\partial u_{n}}{\partial y}+\delta^{2} \frac{\partial v_{n}}{\partial x}\right)+2 \varepsilon \delta \frac{\partial \eta}{\partial x}\left(\frac{\partial v_{n}}{\partial y}-\frac{\partial u_{n}}{\partial x}\right)=0} \\
(\hat{\rho}+\varepsilon r)\left(v_{n}-\frac{\partial \eta}{\partial t}-\varepsilon u_{n} \frac{\partial \eta}{\partial x}\right)=\mu \tau / \cos \theta \\
\kappa \cos \theta\left(\frac{\partial \tau}{\partial y}-\varepsilon \delta^{2} \frac{\partial \eta}{\partial x} \frac{\partial \tau}{\partial x}\right)=-\mu l \tau
\end{gathered}
$$

In all these surface conditions, (A15-A24), $\tan \theta=\varepsilon \delta \partial \eta / \partial x$.

\section{References}

[1] K. R. Atkins, "Third and fourth sound in liquid helium II", Phys. Rev. 113 (1959), 962-965.

[2] K. R. Atkins and I. Rudnick, "Third sound" in Progress in low temperature physics, Vol. 7 (North Holland, Amsterdam, 1970).

[3] D. Bergman, "Hydrodynamics and third sound in thin He II films", Phys. Rev. 188 (1969), 370-384.

[4] D. Bergman, "Third sound in superfluid helium films of arbitrary thickness", Phys. Rev. A3 (1971), 2058-2066.

[5] M. Chester and L. C. Yang, "Superfluid fraction in thin helium films", Phys. Rev. Lett. 31 (1973), 1377-1380.

[6] R. J. Donnelly, Experimental superfludtty (Univ. of Chicago Press, Chicago, 1967).

[7] C. W. F. Everitt, K. R. Atkins and A. Denenstein, "Detection of third sound in liquid helium films", Phys. Rev. Lett. 8 (1962), 161-165.

[8] V L. Ginzburg and A. A. Sobaynin, "Superfluidity of helium II near the $\lambda$-point", Soviet Phys. Uspekhi 19 (1976), 773-812.

[9] R. N. Hills and P. H. Roberts, "On Landau's two-fluid model for helium II", J. Inst. Math. Appl. 9 (1972), 56-67.

[10] R. N. Hills and P. H. Roberts, "Healing and relaxation in flows of helium II-I Generalization of Landau's equations", Internat. J. Engrg. Sci. 15 (1977), 305-316.

[11] R. N. Hills and P. H. Roberts, "On the interface conditions between a liquid and its vapour with application to helium II", J. Non-Equlhb. Thermodyn. 4 (1979), 131-142.

[12] B. A. Huberman, "Superfluid solitons in helium films", Phys. Rev. Lett. 41 (1978), 1389- 1393.

[13] I. M. Khalatnikov, "Absorption and dispersion of sound in a superfluid liquid near the $\lambda$-point", Sovet Phys. JETP 30 (1970), 268-272.

[14] R. S. Johnson, "Shallow water waves on a viscous fluid-the undular bore", Phys. Flurds 15 (1972), 1693-1699.

[15] R. S. Johnson, "On the propagation of long waves on the surface of helium II: linearised theory", Proc. Roy Soc. London Ser. A 362 (1978), 97-111.

[16] R. S. Johnson, "A note on the effect of healing and relaxation in helium II due to heat transfer at a wall”, Rov. Soc. London Ser. A 362 (1978), 375-382

[17] R. S. Johnson, in preparation, 1983.

[18] L. D. Landau, "On a two-fluid model of superfluid helium", J. Phys. 5 (1941), 71-90 (in Russian).

[19] L. D. Landau and E. M. Lifshitz, Fluid mechanics (Pergamon, Oxford, 1959).

[20] S. Nakajima, S. Kurihara and K. Tohdoh, "Soliton in superfluid" He films", Preprint (University of Toyko, 1980). 
[21] K. A. Pickar and K. R. Atkins, "Critical velocity of a superflowing liquid-helium film using third sound", Phys. Rev. 178 (1969), 389-399.

[22] S. J. Putterman, Superfluid hydrodynamics (North-Holland, Amsterdam, 1974).

[23] P. H. Roberts and R. J. Donnelly, "Superfluid mechanics", Annual Rev. Fluid. Mech. 6 (1974), 179-225.

[24] A. A. Sobaynin, "Surface tension of helium II near the $\lambda$-point", Soviet Phys. JETP 34 (1972), 229-232.

[25] G. B. Whitham, "Some comments on wave propagation and shock wave structure with application to magnetohydrodynamics", Comm. Pure Appl. Math. 12 (1959), 113-135.

[26́] G. B. Ẅhitham, Lınear and nonlinear waves (Wiley-Interscience, New York, 1974). 